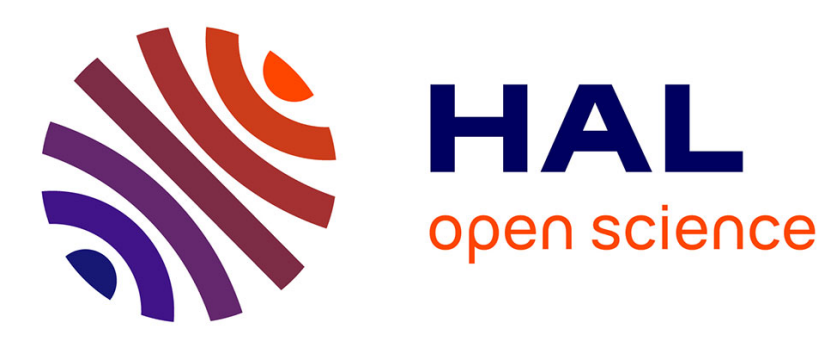

\title{
On the stability of Timoshenko-type systems with internal frictional dampings and discrete time delays
}

Aissa Guesmia, Abdelaziz Soufyane

\section{To cite this version:}

Aissa Guesmia, Abdelaziz Soufyane. On the stability of Timoshenko-type systems with internal frictional dampings and discrete time delays. Applicable Analysis, 2016, 96 (12), pp.2075-2101. 10.1080/00036811.2016.1204439 . hal-02891551

\section{HAL Id: hal-02891551 https://hal.science/hal-02891551}

Submitted on 9 Aug 2020

HAL is a multi-disciplinary open access archive for the deposit and dissemination of scientific research documents, whether they are published or not. The documents may come from teaching and research institutions in France or abroad, or from public or private research centers.
L'archive ouverte pluridisciplinaire HAL, est destinée au dépôt et à la diffusion de documents scientifiques de niveau recherche, publiés ou non, émanant des établissements d'enseignement et de recherche français ou étrangers, des laboratoires publics ou privés. 
archives-ouvertes

\section{On the stability of Timoshenko-type systems with internal frictional dampings and discrete time delays}

Aissa Guesmia, Abdelaziz Soufyane

\section{To cite this version:}

Aissa Guesmia, Abdelaziz Soufyane. On the stability of Timoshenko-type systems with internal frictional dampings and discrete time delays. Applicable Analysis, Taylor \& Francis, 2016, 10.1080/00036811.2016.1204439 . hal-02891551

\section{HAL Id: hal-02891551 \\ https://hal.archives-ouvertes.fr/hal-02891551}

Submitted on 9 Aug 2020

HAL is a multi-disciplinary open access archive for the deposit and dissemination of scientific research documents, whether they are published or not. The documents may come from teaching and research institutions in France or abroad, or from public or private research centers.
L'archive ouverte pluridisciplinaire HAL, est destinée au dépôt et à la diffusion de documents scientifiques de niveau recherche, publiés ou non, émanant des établissements d'enseignement et de recherche français ou étrangers, des laboratoires publics ou privés. 


\title{
On the stability of Timoshenko-type systems with internal frictional dampings and discrete time delays
}

\author{
Aissa Guesmia ${ }^{a}$ and Abdelaziz Soufyane ${ }^{b}$ \\ ${ }^{a}$ Institut Elie Cartan de Lorraine, UMR 7502 Université de Lorraine, Metz Cedex 01, France; ${ }^{b}$ College of Engineering \\ and Applied Sciences, Alhosn University, Abu Dhabi, UAE
}

\begin{abstract}
In this paper, we consider a vibrating system of Timoshenko-type in a bounded one-dimensional domain under Dirichlet-Dirichlet or DirichletNeumann boundary conditions with one or two discrete time delays and one or two internal frictional dampings. First, we show that the system is well posed in the sens of semigroup theory. Second, we prove the exponential stability regardless to the speeds of wave propagation of the system if the weights of the time delays are smaller than the ones of the corresponding dampings, respectively. However, when the weight of one time delay is not smaller than the one of the corresponding damping, we prove the exponential stability in case of equal-speed wave propagation, and the polynomial stability in the opposite case.
\end{abstract}

\section{ARTICLE HISTORY}

Received 10 October 2015

Accepted 16 June 2016

COMMUNICATED BY

G. N'Guerekata

\section{KEYWORDS}

Well posedness; stability; time delay; frictional

damping; Timoshenko-type; semigroup theory; Lyapunov functional

\section{AMS SUBJECT}

CLASSIFICATIONS

35L05; 35L15; 35L70; 93D15

\section{Introduction}

In this paper, we consider the following Timoshenko system:

$$
\left\{\begin{array}{l}
\rho_{1} \varphi_{t t}(x, t)-k_{1}\left(\varphi_{x}+\psi\right)_{x}(x, t)+\lambda_{1} \varphi_{t}(x, t)+\mu_{1} \varphi_{t}\left(x, t-\tau_{1}\right)=0 \\
\rho_{2} \psi_{t t}(x, t)-k_{2} \psi_{x x}(x, t)+k_{1}\left(\varphi_{x}+\psi\right)(x, t)+\lambda_{2} \psi_{t}(x, t)+\mu_{2} \psi_{t}\left(x, t-\tau_{2}\right)=0 \\
\varphi(x, 0)=\varphi_{0}(x), \varphi_{t}(x, 0)=\varphi_{1}(x) \\
\psi(x, 0)=\psi_{0}(x), \psi_{t}(x, 0)=\psi_{1}(x) \\
\varphi_{t}\left(x,-\tau_{1} \rho\right)=f_{1}\left(x,-\tau_{1} \rho\right), \psi_{t}\left(x,-\tau_{2} \rho\right)=f_{2}\left(x,-\tau_{2} \rho\right)
\end{array}\right.
$$

under the Dirichlet-Dirichlet boundary conditions

$$
\varphi(0, t)=\varphi(L, t)=\psi(0, t)=\psi(L, t)=0
$$

or the Dirichlet-Neumann boundary conditions

$$
\varphi(0, t)=\varphi(L, t)=\psi_{x}(0, t)=\psi_{x}(L, t)=0,
$$

for $x \in] 0, L[, t>0, \rho \in] 0,1\left[, \mu_{j} \in \mathbb{R}, L, \rho_{j}, k_{j}, \tau_{j}>0, \lambda_{j} \geq 0(j=1,2)\right.$,

$$
(\varphi, \psi):] 0, L[\times] 0,+\infty\left[\rightarrow \mathbb{R}^{2}\right.
$$


is the state of (1.1) with (1.2) or (1.3),

$$
\left.\varphi_{0}, \varphi_{1}, \psi_{0}, \psi_{1}:\right] 0, L\left[\rightarrow \mathbb{R} \text { and } f_{j}:\right] 0, L[\times]-\tau_{j}, 0[\rightarrow \mathbb{R}
$$

$(j=1,2)$ are given initial data. A subscript $y$ as well as the notation $\partial_{y}$ denote the derivative with respect to $y$. When a function has only one variable, its derivative is noted by $\%$

Our aim is the study of the well posedness and asymptotic behavior of the solutions of (1.1) with (1.2) or (1.3) in case of the equal-speed wave propagation

$$
\frac{k_{1}}{\rho_{1}}=\frac{k_{2}}{\rho_{2}}
$$

as well as in the opposite case.

The Timoshenko-type systems were introduced in [50] to describe the transverse vibration of a beam. Since then, the well posedness and stability of this model has attracted the attention of many researchers using diverse types of dissipative mechanisms. Let us mention here some of these results.

When no time delay is considered (i.e. $\mu_{1}=\mu_{2}=0$ ), investigations showed that the presence of controls (linear or nonlinear feedbacks and/or finite or infinite memories) on both the rotation angle $\varphi$ and the transverse displacement $\psi$ guarantees the stability without any restriction on the constants $\rho_{j}$ and $k_{j}$. However, in the case of only one control, the rate of decay depends heavily on the relation (1.4) and the regularity of the initial data. We quote in this regard [1-3,7,10-16,20,24$30,33,40-42,44,45,48,49]$.

When only one time delay is present (i.e. $\mu_{1} \mu_{2}=0$ and $\left.\left(\mu_{1}, \mu_{2}\right) \neq(0,0)\right)$, the questions related to well posedness and stability/instability of Timoshenko-type systems have attracted considerable attention in recent years and many researchers have shown that the time delay can destabilize a system that was asymptotically stable in the absence of time delay. Under smallness conditions on the weight of the time delay, it was proved that, when the time delay and control are considered on the same equation, the stability still holds; see $[4,8,9,18,19,21,23,43,46]$.

In [47], the stability of Timoshenko systems with two internal time delays and two boundary linear feedbacks was proved under some smallness conditions on $L$ and the weights of the delays.

For the stability of another kind of systems with delay, we refer the reader to $[5,6,17,34-38]$ and the references therein.

As far as we know, the problem of stability of Timoshenko system with two discrete time delays and one or two frictional dampings, as well as the case of one time delay and one frictional damping not considered on the same equation, has never been treated in the literature. Our goal in this paper is to study the well posedness of (1.1) with (1.2) or (1.3) and investigate the effect of presence of one or two frictional dampings on the asymptotic behavior of their solutions. When the weight of one time delay is not smaller than the one of the corresponding damping, the system (1.1) with (1.2) or (1.3) is not necessarily dissipative with respect to its classical energy, so some new difficulties are generated.

The proof of the well posedness is based on the maximal monotone operators and semigroup approach. However, the proof of stability estimates is based on the multiplier method. The paper is organized as follows. Section 2 deals with the well posedness of (1.1) with (1.2) or (1.3). In section 3, we present our exponential and polynomial stability results. In section 4 , we prove the exponential stability in both cases (1.2) and (1.3), when the weights of the time delays are smaller than the ones of the corresponding dampings, respectively. After, we consider the case where the weight of one time delay is not smaller than the one of the corresponding damping and we prove in sections 5 and 6 , respectively, the exponential stability in case (1.4), and the polynomial stability in the opposite case. Finally, we conclude in Section 7 by some remarks and open questions. 


\section{Well posedness}

In this section, we prove the existence, uniqueness, and smoothness of the solution of (1.1) with (1.2) or (1.3). For this purpose, we adopt the technique of [34], (see also [35-38]) to reformulate (1.1) with (1.2) or (1.3) in the first-order system (2.3) below and prove that the operator $\mathscr{A}+\mathscr{B}$ defined, respectively, in (2.4) and (2.6) generates a contraction semigroup on the Hilbert space $\mathscr{H}$ given in (2.7).

Let us consider the following new variables:

$$
\left\{\begin{array}{l}
\left.z_{1}(x, \rho, t):=\varphi_{t}\left(x, t-\tau_{1} \rho\right), \text { in }\right] 0, L[\times] 0,1[\times] 0,+\infty[ \\
\left.z_{2}(x, \rho, t):=\psi_{t}\left(x, t-\tau_{2} \rho\right), \text { in }\right] 0, L[\times] 0,1[\times] 0,+\infty[
\end{array}\right.
$$

Then it is easy to check that

$$
\begin{cases}\tau_{j} z_{j t}(x, \rho, t)+z_{j \rho}(x, \rho, t)=0, & \text { in }] 0, L[\times] 0,1[\times] 0,+\infty[ \\ z_{1}(x, 0, t)=\varphi_{t}(x, t), z_{2}(x, 0, t)=\psi_{t}(x, t), & \text { in }] 0, L[\times] 0,+\infty[\end{cases}
$$

Now, we present a short discussion of the formulation of (1.1) with (1.2) or (1.3) in a first-order system. For this purpose, let

$$
\mathscr{U}:=\left(\varphi, \varphi_{t}, \psi, \psi_{t}, z_{1}, z_{2}\right)^{T} \quad \text { and } \quad \mathscr{U}_{0}:=\left(\varphi_{0}, \varphi_{1}, \psi_{0}, \psi_{1}, f_{1}\left(.,-\tau_{1} .\right), f_{2}\left(.,-\tau_{2} .\right)\right)^{T}
$$

then $\mathscr{U}$ satisfies the problem

$$
\left\{\begin{array}{l}
\mathscr{U}^{\prime}(t)=(\mathscr{A}+\mathscr{B}) \mathscr{U}(t), \quad t>0 \\
\mathscr{U}(0)=\mathscr{U}_{0}
\end{array}\right.
$$

where the operators $\mathscr{B}$ and $\mathscr{A}$ are defined by

$$
\begin{gathered}
\mathscr{B}\left(u_{1}, u_{2}, v_{1}, v_{2}, z_{1}, z_{2}\right)^{T}=\left(0, \frac{\xi_{1}^{0}}{\rho_{1}} u_{2}, 0, \frac{\xi_{2}^{0}}{\rho_{2}} v_{2}, 0,0\right)^{T}, \\
\xi_{j}^{0}= \begin{cases}0 & \text { if }\left|\mu_{j}\right| \leq \lambda_{j}, \\
\left|\mu_{j}\right| & \text { if }\left|\mu_{j}\right|>\lambda_{j}\end{cases}
\end{gathered}
$$

and

$$
\mathscr{A}\left(\begin{array}{c}
u_{1} \\
u_{2} \\
v_{1} \\
v_{2} \\
w_{1} \\
w_{2}
\end{array}\right)=\left(\begin{array}{c}
u_{2} \\
\frac{k_{1}}{\rho_{1}}\left(u_{1 x x}+v_{1 x}\right)-\frac{\lambda_{1}+\xi_{1}^{0}}{\rho_{1}} u_{2}-\frac{\mu_{1}}{\rho_{1}} w_{1}(1) \\
v_{2} \\
\frac{k_{2}}{\rho_{2}} v_{1 x x}-\frac{k_{1}}{\rho_{2}}\left(u_{1 x}+v_{1}\right)-\frac{\lambda_{2}+\xi_{2}^{0}}{\rho_{2}} v_{2}-\frac{\mu_{2}}{\rho_{2}} w_{2}(1) \\
\frac{-1}{\tau_{1}} w_{1 \rho} \\
\frac{-1}{\tau_{2}} w_{2 \rho}
\end{array}\right)
$$

with domains $D(\mathscr{B})=\mathscr{H}$ and

$$
\begin{aligned}
D(\mathscr{A}) & =\left\{\left(u_{1}, u_{2}, v_{1}, v_{2}, w_{1}, w_{2}\right)^{T} \in H:\right. \\
\left(w_{1 \rho}, w_{2 \rho}\right) & \left.\in L^{2}(] 0,1\left[, L^{2}(] 0, L[)\right) \times L^{2}(] 0,1\left[, V_{0}\right),\left(w_{1}(0), w_{2}(0)\right)=\left(u_{2}, v_{2}\right)\right\},
\end{aligned}
$$


where

$$
\begin{aligned}
H:= & \left(H^{2}(] 0, L[) \cap H_{0}^{1}(] 0, L[)\right) \times H_{0}^{1}(] 0, L[) \times\left(H_{*}^{2}(] 0, L[) \cap V_{1}\right) \\
& \times V_{1} \times L^{2}(] 0,1\left[, L^{2}(] 0, L[)\right) \times L^{2}(] 0,1\left[, V_{0}\right),
\end{aligned}
$$

$\mathscr{H}$ is the energy space defined by

$$
\begin{gathered}
\mathscr{H}:=H_{0}^{1}(] 0, L[) \times L^{2}(] 0, L[) \times V_{1} \times V_{0} \times L^{2}(] 0,1\left[, L^{2}(] 0, L[)\right) \times L^{2}(] 0,1\left[, V_{0}\right), \\
H_{*}^{2}(] 0, L[)= \begin{cases}H^{2}(] 0, L[), & \text { in case }(1.2), \\
\left.v \in H^{2}(] 0, L[), \partial_{x} v(0)=\partial_{x} v(L)=0\right\}, & \text { in case }(1.3),\end{cases} \\
V_{1}= \begin{cases}H_{0}^{1}(] 0, L[), & \text { in case }(1.2), \\
\left.v \in H^{1}(] 0, L[), \int_{0}^{L} v \mathrm{~d} x=0\right\}, & \text { in case }(1.3)\end{cases}
\end{gathered}
$$

and

$$
V_{0}= \begin{cases}L^{2}(] 0, L[), & \text { in case (1.2) } \\ \left\{v \in L^{2}(] 0, L[), \int_{0}^{L} v \mathrm{~d} x=0\right\}, & \text { in case (1.3) }\end{cases}
$$

For $U=\left(u_{1}, u_{2}, v_{1}, v_{2}, w_{1}, w_{2}\right)^{T}, \bar{U}=\left(\bar{u}_{1}, \bar{u}_{2}, \bar{v}_{1}, \bar{v}_{2}, \bar{w}_{1}, \bar{w}_{2}\right)^{T}$ and

$$
\xi_{j}= \begin{cases}\tau_{j} \lambda_{j} & \text { if } 0<\left|\mu_{j}\right| \leq \lambda_{j} \\ \tau_{j}\left|\mu_{j}\right| & \text { if }\left|\mu_{j}\right|>\lambda_{j} \text { or } \mu_{j}=0\end{cases}
$$

the space $L^{2}(] 0,1\left[, L^{2}(] 0, L[)\right) \times L^{2}(] 0,1\left[, V_{0}\right)$ endowed with the inner product

$$
\begin{aligned}
& \left\langle\left(w_{1}, w_{2}\right),\left(\bar{w}_{1}, \bar{w}_{2}\right)\right\rangle_{L^{2}(] 0,1\left[, L^{2}(] 0, L[)\right) \times L^{2}(] 0,1\left[, V_{0}\right)} \\
& =\int_{0}^{L} \int_{0}^{1}\left(\xi_{1} w_{1}(x, \rho) \bar{w}_{1}(x, \rho)+\xi_{2} w_{2}(x, \rho) \bar{w}_{2}(x, \rho)\right) \mathrm{d} \rho \mathrm{d} x
\end{aligned}
$$

is a Hilbert space, and we define the inner product in $\mathscr{H}$ as follows:

$$
\begin{aligned}
\langle U, \bar{U}\rangle_{\mathscr{H}}= & \int_{0}^{L}\left(\rho_{1} u_{2} \bar{u}_{2}+\rho_{2} v_{2} \bar{v}_{2}+k_{1}\left(u_{1 x}+v_{1}\right)\left(\bar{u}_{1 x}+\bar{v}_{1}\right)+k_{2} v_{1 x} \bar{v}_{1 x}\right) \mathrm{d} x \\
& +\left\langle\left(w_{1}, w_{2}\right),\left(\bar{w}_{1}, \bar{w}_{2}\right)\right\rangle_{L^{2}(] 0,1\left[L^{2}(] 0, L[)\right) \times L^{2}(] 0,1\left[, V_{0}\right)} .
\end{aligned}
$$

\section{Remark 2.1:}

(1) If $\mu_{j}=0$, the variable $z_{j}$ is not considered, and therefore, the corresponding components in the definition of $\mathscr{U}, \mathscr{U} 0, \mathscr{B}, \mathscr{A}, D(\mathscr{A}), H$, and $\mathscr{H}$ will not appear.

(2) Let $c_{0}$ be the smallest positive constant depending only on $L$ and satisfying (Poincarés inequality)

$$
\int_{0}^{L} v^{2} \mathrm{~d} x \leq c_{0} \int_{0}^{L} v_{x}^{2} \mathrm{~d} x, \quad \forall v \in H_{*}^{1}(] 0, L[)
$$


where

$$
H_{*}^{1}(] 0, L[)=\left\{v \in H^{1}(] 0, L[), v(0)=v(L)=0 \text { or } \int_{0}^{L} v \mathrm{~d} x=0\right\} .
$$

By applying (2.12) on $v_{1}$, we see that

$$
\begin{aligned}
\int_{0}^{L}\left(u_{1 x}^{2}+v_{1 x}^{2}\right) \mathrm{d} x & \leq \int_{0}^{L}\left(2\left(u_{1 x}+v_{1}\right)^{2}+2 v_{1}^{2}+v_{1 x}^{2}\right) \mathrm{d} x \\
& \leq \int_{0}^{L}\left(2\left(u_{1 x}+v_{1}\right)^{2}+\left(2 c_{0}+1\right) v_{1 x}^{2}\right) \mathrm{d} x \\
& \leq \max \left\{\frac{2}{k_{1}}, \frac{2 c_{0}+1}{k_{2}}\right\} \int_{0}^{L}\left(k_{1}\left(u_{1 x}+v_{1}\right)^{2}+k_{2} v_{1 x}^{2}\right) \mathrm{d} x
\end{aligned}
$$

Because $\int_{0}^{L} u_{1 x}^{2} \mathrm{~d} x$ and $\int_{0}^{L} v_{1 x}^{2} \mathrm{~d} x$ define norms, for $u_{1}$ and $v_{1}$ on $H_{0}^{1}(] 0, L[)$ and $V_{1}$, respectively, then

$$
\int_{0}^{L}\left(k_{1}\left(u_{1 x}+v_{1}\right)\left(\bar{u}_{1 x}+\bar{v}_{1}\right)+k_{2} v_{1 x} \bar{v}_{1 x}\right) \mathrm{d} x
$$

generates a norm on $H_{0}^{1}(] 0, L[) \times V_{1}$, for $\left(u_{1}, v_{1}\right)$, equivalent to the one induced by $\left(H^{1}(] 0, L[)\right)^{2}$. Consequently, $\mathscr{H}$ is a Hilbert space.

(3) In case of Dirichlet-Neumann boundary conditions (1.3), Poincarés inequality (2.12) is not necessarily applicable for $\psi$. To overcome this problem, let us consider

$$
\begin{gathered}
g(t)=\int_{0}^{L} \psi(x, t) \mathrm{d} x(\text { for } t>0), \quad g_{-1}(t)=\int_{0}^{L} f_{2}\left(x, t-\tau_{2}\right) \mathrm{d} x(\text { for } t \in] 0, \tau_{2}[), \\
g_{-1}^{0}=\int_{0}^{L} \psi_{0}(x) \mathrm{d} x \text { and } g_{-1}^{1}=\int_{0}^{L} \psi_{1}(x) \mathrm{d} x .
\end{gathered}
$$

Using the second equation and initial data $\left(\psi_{0}, \psi_{1}, f_{2}\right)$ in (1.1) and the boundary conditions (1.3), we easily verify that

$$
\begin{cases}\rho_{2} g^{\prime \prime}(t)+\lambda_{2} g^{\prime}(t)+k_{1} g(t)+\mu_{2} g^{\prime}\left(t-\tau_{2}\right)=0, & t>0, \\ g^{\prime}(t)=\int_{0}^{L} f_{2}(x, t) \mathrm{d} x, & t \in]-\tau_{2}, 0[, \\ g(0)=g_{-1}^{0}, \quad g^{\prime}(0)=g_{-1}^{1} . & \end{cases}
$$

In particular,

$$
\left\{\begin{array}{l}
\left.\left.\rho_{2} g^{\prime \prime}(t)+\lambda_{2} g^{\prime}(t)+k_{1} g(t)+\mu_{2} g_{-1}(t)=0, t \in\right] 0, \tau_{2}\right] \\
g(0)=g_{-1}^{0}, \quad g^{\prime}(0)=g_{-1}^{1}
\end{array}\right.
$$

The characteristic equation $\rho_{2} s^{2}+\lambda_{2} s+k_{1}=0$ of the homogeneous equation associated to (2.15) has the solutions

$$
\begin{cases}s_{0}=\frac{-\lambda_{2}}{2 \rho_{2}} & \text { if } \lambda_{2}=2 \sqrt{k_{1} \rho_{2}}, \\ s_{1}=\frac{-\lambda_{2}-\sqrt{\left|\lambda_{2}^{2}-4 k_{1} \rho_{2}\right|}}{2 \rho_{2}}, s_{2}=\frac{-\lambda_{2}+\sqrt{\left|\lambda_{2}^{2}-4 k_{1} \rho_{2}\right|}}{2 \rho_{2}} & \text { if } \lambda_{2}>2 \sqrt{k_{1} \rho_{2}} \\ s_{0}-\left(s_{2}-s_{0}\right) i, s_{0}+\left(s_{2}-s_{0}\right) i & \text { if } \lambda_{2} \in\left[0,2 \sqrt{k_{1} \rho_{2}}[\right.\end{cases}
$$


(here $i$ is the imaginary number satisfying $i^{2}=-1$ ). Then, using classical arguments, we find that the unique solution $g_{0}$ of $(2.15)$ is given by $g_{0}=g_{0}^{0}+g_{0}^{1}$, where

$$
g_{0}^{0}(t)= \begin{cases}\left(\left(g_{-1}^{1}-s_{0} g_{-1}^{0}\right) t+g_{-1}^{0}\right) e^{s_{0} t} & \text { if } \lambda_{2}=2 \sqrt{k_{1} \rho_{2}}, \\ \frac{1}{s_{2}-s_{1}}\left(s_{2} g_{-1}^{0}-g_{-1}^{1}\right) e^{s_{1} t}+\frac{1}{s_{2}-s_{1}}\left(g_{-1}^{1}-s_{1} g_{-1}^{0}\right) e^{s_{2} t} & \text { if } \lambda_{2}>2 \sqrt{k_{1} \rho_{2}}, \\ \left(g_{-1}^{0} \cos \left(\left(s_{2}-s_{0}\right) t\right)+\frac{1}{s_{2}-s_{0}}\left(g_{-1}^{1}-s_{0} g_{-1}^{0}\right) \sin \left(\left(s_{2}-s_{0}\right) t\right)\right) e^{s_{0} t} & \text { if } \lambda_{2} \in\left[0,2 \sqrt{k_{1} \rho_{2}}[\right.\end{cases}
$$

and

$$
g_{0}^{1}(t)= \begin{cases}-\frac{\mu_{2} e^{s_{0} t}}{\rho_{2}} \int_{0}^{t} \int_{0}^{s} g_{-1}(\tau) e^{-s_{0} \tau} \mathrm{d} \tau \mathrm{d} s & \text { if } \lambda_{2}=2 \sqrt{k_{1} \rho_{2}}, \\ -\frac{\mu_{2} e^{s_{1}} t}{\rho_{2}} \int_{0}^{t} e^{2\left(s_{0}-s_{1}\right) s} \int_{0}^{s} g_{-1}(\tau) e^{-\left(2 s_{0}-s_{1}\right) \tau} \mathrm{d} \tau \mathrm{d} s & \text { if } \lambda_{2}>2 \sqrt{k_{1} \rho_{2}}, \\ -\frac{\mu_{2}}{\rho_{2}} \operatorname{Re}\left(e^{\left(s_{0}-i\left(s_{2}-s_{0}\right)\right) t} \int_{0}^{t} e^{2 i\left(s_{2}-s_{0}\right) s} \int_{0}^{s} g_{-1}(\tau) e^{-\left(s_{0}+i\left(s_{2}-s_{0}\right)\right) \tau} \mathrm{d} \tau \mathrm{d} s\right) & \text { if } \lambda_{2} \in\left[0,2 \sqrt{k_{1} \rho_{2}}[\right.\end{cases}
$$

where Re denotes the real part. Therefore, for any $n \in \mathbb{N}^{*}$, the unique solution $g_{n}$ of

$$
\left\{\begin{array}{l}
\left.\left.\rho_{2} g^{\prime \prime}(t)+\lambda_{2} g^{\prime}(t)+k_{1} g(t)+\mu_{2} g_{n-1}(t)=0, \quad t \in\right] n \tau_{2},(n+1) \tau_{2}\right] \\
g\left(n \tau_{2}\right)=g_{n-1}\left(n \tau_{2}\right), \quad g^{\prime}\left(n \tau_{2}\right)=g_{n-1}^{\prime}\left(n \tau_{2}\right)
\end{array}\right.
$$

is defined as $g_{0}$ with $g_{-1}, g_{-1}^{0}, g_{-1}^{1}, \int_{0}^{t}$ and $\int_{0}^{s}$ are replaced by $g_{n-1}, g_{n-1}\left(n \tau_{2}\right), g_{n-1}^{\prime}\left(n \tau_{2}\right), \int_{n \tau_{2}}^{t}$ and $\int_{n \tau_{2}}^{s}$, respectively. Consequently, the unique two times differential solution $g$ of $(2.14)$ is given by

$$
\begin{cases}g(t)=g_{n}(t), & \left.t \in] n \tau_{2},(n+1) \tau_{2}\right], n \in \mathbb{N}, \\ g\left(n \tau_{2}\right)=g_{n-1}\left(n \tau_{2}\right), \quad g^{\prime}\left(n \tau_{2}\right)=g_{n-1}^{\prime}\left(n \tau_{2}\right), n \in \mathbb{N}^{*} & \\ g(0)=g_{0}^{0}, \quad g^{\prime}(0)=g_{0}^{1} & \end{cases}
$$

Now, we put

$$
\tilde{\psi}=\psi-\frac{1}{L} g
$$

Then, one can easily check that

$$
\int_{0}^{L} \tilde{\psi} \mathrm{d} x=0
$$

and, hence, Poincaré's inequality (2.12) is applicable for $\tilde{\psi}$ provided that $\tilde{\psi} \in H^{1}(] 0, L[)$. In addition, $(\varphi, \tilde{\psi})$ satisfies (1.1) with (1.3) and initial data

$$
\tilde{\psi}_{0}=\psi_{0}-\frac{1}{L} \int_{0}^{L} \psi_{0} \mathrm{~d} x, \quad \tilde{\psi}_{1}=\psi_{1}-\frac{1}{L} \int_{0}^{L} \psi_{1} \mathrm{~d} x \quad \text { and } \quad \tilde{f}_{2}=f_{2}-\frac{1}{L} \int_{0}^{L} f_{2} \mathrm{~d} x
$$

instead of $\psi_{0}, \psi_{1}$, and $f_{2}$, respectively. In the sequel, we work with $\tilde{\psi}$ instead of $\psi$ when (1.3) holds, but, for simplicity of notation, we use always $\psi$ instead of $\tilde{\psi}$.

Theorem 2.2: $\quad$ For any $\mathscr{U}_{0} \in \mathscr{H}$, there exists a unique solution

$$
\mathscr{U} \in C\left(\mathbb{R}^{+}, \mathscr{H}\right)
$$

of problem (2.3). Moreover, if $\mathscr{U}_{0} \in D(\mathscr{A})$, then

$$
\mathscr{U} \in C\left(\mathbb{R}^{+}, D(\mathscr{A})\right) \cap C^{1}\left(\mathbb{R}^{+}, \mathscr{H}\right) .
$$


Proof: In order to prove the result stated in Theorem 2.2, we will use the semigroup approach; that is, we will show that the operator $\mathscr{A}+\mathscr{B}$ generates a $C_{0}$-semigroup in $\mathscr{H}$. In this step, we concern ourselves to prove that the operator $\mathscr{A}$ is dissipative. Indeed, exploiting (2.2), (2.6) and the definition of $\langle,\rangle_{\mathscr{C}}$, integrating by parts with respect to $x$ and using the boundary conditions (1.2) or (1.3), we have, for $U=\left(u_{1}, u_{2}, v_{1}, v_{2}, w_{1}, w_{2}\right)^{T} \in D(\mathscr{A})$,

$$
\begin{aligned}
\langle\mathscr{A} U, U\rangle_{\mathscr{H}}= & -\left(\lambda_{1}+\xi_{1}^{0}\right) \int_{0}^{L} u_{2}^{2} \mathrm{~d} x-\left(\lambda_{2}+\xi_{2}^{0}\right) \int_{0}^{L} v_{2}^{2} \mathrm{~d} x-\mu_{1} \int_{0}^{L} w_{1}(x, 1) u_{2} \mathrm{~d} x \\
& -\mu_{2} \int_{0}^{L} w_{2}(x, 1) v_{2} \mathrm{~d} x-\frac{\xi_{1}}{\tau_{1}} \int_{0}^{L} \int_{0}^{1} w_{1}(x, \rho) w_{1 \rho}(x, \rho) \mathrm{d} \rho \mathrm{d} x \\
& -\frac{\xi_{2}}{\tau_{2}} \int_{0}^{L} \int_{0}^{1} w_{2}(x, \rho) w_{2} \rho(x, \rho) \mathrm{d} \rho \mathrm{d} x .
\end{aligned}
$$

Looking now at the last four integrals of the right-hand side of (2.18), we have

$$
\begin{aligned}
- & \frac{\xi_{1}}{\tau_{1}} \int_{0}^{L} \int_{0}^{1} w_{1}(x, \rho) w_{1 \rho}(x, \rho) \mathrm{d} \rho \mathrm{d} x-\frac{\xi_{2}}{\tau_{2}} \int_{0}^{L} \int_{0}^{1} w_{2}(x, \rho) w_{2 \rho}(x, \rho) \mathrm{d} \rho \mathrm{d} x \\
= & -\frac{\xi_{1}}{\tau_{1}} \int_{0}^{L} \int_{0}^{1} \frac{1}{2} \frac{\partial}{\partial \rho} w_{1}^{2}(x, \rho) \mathrm{d} \rho \mathrm{d} x-\frac{\xi_{2}}{\tau_{2}} \int_{0}^{L} \int_{0}^{1} \frac{1}{2} \frac{\partial}{\partial \rho} w_{2}^{2}(x, \rho) \mathrm{d} \rho \mathrm{d} x \\
& =\frac{\xi_{1}}{2 \tau_{1}} \int_{0}^{L}\left(w_{1}^{2}(x, 0)-w_{1}^{2}(x, 1)\right) \mathrm{d} x+\frac{\xi_{2}}{2 \tau_{2}} \int_{0}^{L}\left(w_{2}^{2}(x, 0)-w_{2}^{2}(x, 1)\right) \mathrm{d} x
\end{aligned}
$$

and, using Young's inequality,

$$
\begin{aligned}
- & \mu_{1} \int_{0}^{L} w_{1}(x, 1) u_{2} \mathrm{~d} x-\mu_{2} \int_{0}^{L} w_{2}(x, 1) v_{2} \mathrm{~d} x \leq \frac{\left|\mu_{1}\right|}{2} \int_{0}^{L}\left(u_{2}^{2}+w_{1}^{2}(x, 1)\right) \mathrm{d} x+\frac{\left|\mu_{2}\right|}{2} \\
& \int_{0}^{L}\left(v_{2}^{2}+w_{2}^{2}(x, 1)\right) \mathrm{d} x .
\end{aligned}
$$

Consequently, because $w_{1}^{2}(x, 0)=u_{2}^{2}(x)$ and $w_{2}^{2}(x, 0)=v_{2}^{2}(x),(2.18)$ becomes

$$
\begin{aligned}
\langle\mathscr{A} U, U\rangle_{\mathscr{H}} \leq & \left(\frac{\xi_{1}}{2 \tau_{1}}+\frac{\left|\mu_{1}\right|}{2}-\lambda_{1}-\xi_{1}^{0}\right) \int_{0}^{L} u_{2}^{2} \mathrm{~d} x+\left(\frac{\xi_{2}}{2 \tau_{2}}+\frac{\left|\mu_{2}\right|}{2}-\lambda_{2}-\xi_{2}^{0}\right) \int_{0}^{L} v_{2}^{2} \mathrm{~d} x \\
& +\left(\frac{\left|\mu_{1}\right|}{2}-\frac{\xi_{1}}{2 \tau_{1}}\right) \int_{0}^{L} w_{1}^{2}(x, 1) \mathrm{d} x+\left(\frac{\left|\mu_{2}\right|}{2}-\frac{\xi_{2}}{2 \tau_{2}}\right) \int_{0}^{L} w_{2}^{2}(x, 1) \mathrm{d} x .
\end{aligned}
$$

Using (2.5) and (2.11), we get

$$
\frac{\xi_{j}}{2 \tau_{j}}+\frac{\left|\mu_{j}\right|}{2}-\lambda_{j}-\xi_{j}^{0} \leq 0 \text { and } \frac{\left|\mu_{j}\right|}{2}-\frac{\xi_{j}}{2 \tau_{j}} \leq 0 .
$$

Hence, we deduce from (2.19) that $\langle\mathscr{A} U, U\rangle_{\mathscr{H}} \leq 0$, and consequently, the operator $\mathscr{A}$ is dissipative.

Now, we show that the operator $I d-\mathscr{A}$ is surjective. Indeed, given

$$
U_{0}=\left(\phi_{1}, \phi_{2}, g_{1}, g_{2}, h_{1}, h_{2}\right)^{T} \in \mathscr{H},
$$


we seek $U=\left(u_{1}, u_{2}, v_{1}, v_{2}, w_{1}, w_{2}\right)^{T} \in D(\mathscr{A})$ solution of $(I d-\mathscr{A}) U=U_{0}$; that is,

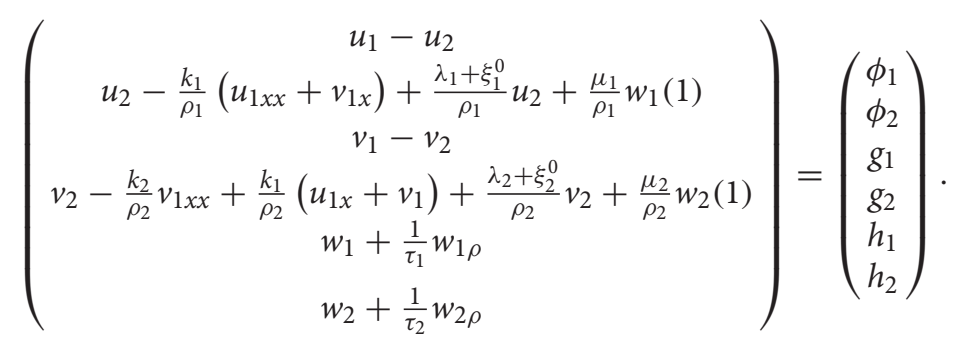

Suppose that we have found

$$
\left(u_{1}, v_{1}\right) \in\left(H^{2}(] 0, L[) \cap H_{0}^{1}(] 0, L[)\right) \times\left(H_{*}^{2}(] 0, L[) \cap V_{1}\right) .
$$

Then the first and third equations in (2.20) give

$$
\left\{\begin{array}{l}
u_{2}=u_{1}-\phi_{1} \\
v_{2}=v_{1}-g_{1}
\end{array}\right.
$$

It is clear that $\left(u_{2}, v_{2}\right) \in H_{0}^{1}(] 0, L[) \times V_{1}$. Furthermore,

$$
\left\{\begin{array}{l}
w_{1}(x, \rho)=\left(u_{1}(x)-\phi_{1}(x)\right) e^{-\tau_{1} \rho}+\tau_{1} e^{-\tau_{1} \rho} \int_{0}^{\rho} h_{1}(x, \sigma) e^{\tau_{1} \sigma} \mathrm{d} \sigma \\
w_{2}(x, \rho)=\left(v_{1}(x)-g_{1}(x)\right) e^{-\tau_{2} \rho}+\tau_{2} e^{-\tau_{2} \rho} \int_{0}^{\rho} h_{2}(x, \sigma) e^{\tau_{2} \sigma} \mathrm{d} \sigma
\end{array}\right.
$$

satisfy the last two equations in (2.20),

$$
\left(w_{1}, w_{2}\right),\left(w_{1 \rho}, w_{2 \rho}\right) \in L^{2}(] 0,1\left[, L^{2}(] 0, L[)\right) \times L^{2}(] 0,1\left[, V_{0}\right)
$$

and, according to $(2.22),\left(w_{1}(0), w_{2}(0)\right)=\left(u_{2}, v_{2}\right)$. Puting

$$
\left\{\begin{array}{l}
w_{1}^{0}(x):=-\phi_{1}(x) e^{-\tau_{1}}+\tau_{1} e^{-\tau_{1}} \int_{0}^{1} h_{1}(x, \sigma) e^{\tau_{1} \sigma} \mathrm{d} \sigma \\
w_{2}^{0}(x):=-g_{1}(x) e^{-\tau_{2}}+\tau_{2} e^{-\tau_{2}} \int_{0}^{1} h_{2}(x, \sigma) e^{\tau_{2} \sigma} \mathrm{d} \sigma
\end{array}\right.
$$

We deduce from (2.23) that

$$
\left\{\begin{array}{l}
w_{1}(x, 1)=e^{-\tau_{1}} u_{1}(x)+w_{1}^{0}(x), \\
w_{2}(x, 1)=e^{-\tau_{2}} v_{1}(x)+w_{2}^{0}(x) .
\end{array}\right.
$$

By using (2.22) and (2.25), the second and fourth equations in (2.20) are equivalent to

$$
\left\{\begin{array}{l}
\left(1+\frac{\lambda_{1}+\mu_{1} e^{-\tau_{1}}+\xi_{1}^{0}}{\rho_{1}}\right) u_{1}-\frac{k_{1}}{\rho_{1}}\left(u_{1 x x}+v_{1 x}\right)=\phi_{2}+\left(1+\frac{\lambda_{1}+\xi_{1}^{0}}{\rho_{1}}\right) \phi_{1}-\frac{\mu_{1}}{\rho_{1}} w_{1}^{0}, \\
\left(1+\frac{\lambda_{2}+\mu_{2} e^{-\tau_{2}}+\xi_{2}^{0}}{\rho_{2}}\right) v_{1}-\frac{k_{2}}{\rho_{2}} v_{1 x x}+\frac{k_{1}}{\rho_{2}}\left(u_{1 x}+v_{1}\right)=g_{2}+\left(1+\frac{\lambda_{2}+\xi_{2}^{0}}{\rho_{2}}\right) g_{1}-\frac{\mu_{2}}{\rho_{2}} w_{2}^{0} .
\end{array}\right.
$$

So, proving that $I d-\mathscr{A}$ is surjective is reduced to prove that (2.26) has at least one solution satisfying (2.21). To do so, multiplying the first and second equations in (2.26) by $\rho_{1} l_{1}$ and $\rho_{2} l_{2}$, respectively, 
where $\left(l_{1}, l_{2}\right) \in H_{0}^{1}(] 0, L[) \times V_{1}$, and integrating by parts with respect to $x$, we see that any solution (2.21) of (2.26) satisfies the variational formulation

$$
\mathscr{R}\left(\left(u_{1}, v_{1}\right),\left(l_{1}, l_{2}\right)\right)=\mathscr{L}\left(l_{1}, l_{2}\right), \quad \forall\left(l_{1}, l_{2}\right) \in H_{0}^{1}(] 0, L[) \times V_{1},
$$

where the bilinear form $\mathscr{R}:\left(H_{0}^{1}(] 0, L[) \times V_{1}\right)^{2} \rightarrow \mathbb{R}$ and the linear form $\mathscr{L}: H_{0}^{1}(] 0, L[) \times V_{1} \rightarrow \mathbb{R}$ are defined by

$$
\begin{aligned}
\mathscr{R}\left((\varphi, \psi),\left(w_{1}, w_{2}\right)\right)= & \int_{0}^{L}\left(\left(\rho_{1}+\lambda_{1}+\mu_{1} e^{-\tau_{1}}+\xi_{1}^{0}\right) u_{1} l_{1}+\left(\rho_{2}+\lambda_{2}+\mu_{2} e^{-\tau_{2}}+\xi_{2}^{0}\right) v_{1} l_{2}\right) \mathrm{d} x \\
& +\int_{0}^{L}\left(k_{1}\left(u_{1 x}+v_{1}\right)\left(l_{1 x}+l_{2}\right)+k_{2} v_{1 x} l_{2 x}\right) \mathrm{d} x
\end{aligned}
$$

and

$\mathscr{L}\left(l_{1}, l_{2}\right)=\int_{0}^{L}\left(\left(\rho_{1} \phi_{2}+\left(\rho_{1}+\lambda_{1}+\xi_{1}^{0}\right) \phi_{1}-\mu_{1} w_{1}^{0}\right) l_{1}+\left(\rho_{2} g_{2}+\left(\rho_{2}+\lambda_{2}+\xi_{2}^{0}\right) g_{1}-\mu_{2} w_{2}^{0}\right) l_{2}\right) \mathrm{d} x$.

It is easy to verify that $\mathscr{R}$ is continuous and coercive, and $\mathscr{L}$ is continuous. So applying the Lax-Milgram theorem, we deduce that problem (2.27) admits a unique solution

$$
\left(u_{1}, v_{1}\right) \in H_{0}^{1}(] 0, L[) \times V_{1} .
$$

Applying the classical elliptic regularity arguments, it follows that $\left(u_{1}, v_{1}\right)$ satisfies $(2.21)$. Therefore, the operator $I d-\mathscr{A}$ is surjective.

Since $\mathscr{A}$ is dissipative and $I d-\mathscr{A}$ is surjective, $\mathscr{A}$ is maximal monotone. Therefore, using LummerPhillips theorem (see [39]), we deduce that $\mathscr{A}$ is an infinitesimal generator of a linear contraction $C_{0}$-semigroup on $\mathscr{H}$. On the other hand, we see that the linear operator $\mathscr{B}$ is Lipschitz continuous. So, finally, also $\mathscr{A}+\mathscr{B}$ is an infinitesimal generator of a linear contraction $C_{0}$-semigroup on $\mathscr{H}$ (see [39]: Ch. 3 - Theorem 1.1). Consequently, the well-posedness results of Theorem 2.2 follow from the Hille-Yosida theorem (see [22] and [39]).

\section{Stability}

To announce our stability results, we consider the energy functional associated with (1.1) and the boundary conditions (1.2) or (1.3) defined by

$$
\begin{aligned}
E(t):= & \frac{1}{2}\|\mathscr{U}(t)\|_{\mathscr{H}}^{2} \\
= & \frac{1}{2} \int_{0}^{L}\left(\rho_{1} \varphi_{t}^{2}+\rho_{2} \psi_{t}^{2}+k_{1}\left(\varphi_{x}+\psi\right)^{2}+k_{2} \psi_{x}^{2}\right) \mathrm{d} x \\
& +\frac{\xi_{1}}{2} \int_{0}^{L} \int_{0}^{1} \varphi_{t}^{2}\left(x, t-\tau_{1} \rho\right) \mathrm{d} \rho \mathrm{d} x+\frac{\xi_{2}}{2} \int_{0}^{L} \int_{0}^{1} \psi_{t}^{2}\left(x, t-\tau_{2} \rho\right) \mathrm{d} \rho \mathrm{d} x .
\end{aligned}
$$

Now, independently of (1.4) and in both cases (1.2) and (1.3), we give our first stability result which concerns the case

$$
\left|\mu_{1}\right|<\lambda_{1} \text { and }\left|\mu_{2}\right|<\lambda_{2}
$$


Theorem 3.1: Assume that (3.2) is satisfied and let $\mathscr{U}_{0} \in \mathscr{H}$. Then there exist positive constants $c_{1}$ and $c_{2}$ for which E satisfies

$$
E(t) \leq c_{2} e^{-c_{1} t}, \quad \forall t \geq 0
$$

Our second stability result concerns the case when (1.4) and one of the following situations hold:

$$
\begin{gathered}
\left|\mu_{1}\right|=\lambda_{1} \quad \text { and }\left|\mu_{2}\right|<\lambda_{2}, \\
\left|\mu_{1}\right|<\lambda_{1} \text { and }\left|\mu_{2}\right|=\lambda_{2}, \\
\left|\mu_{1}\right|>\lambda_{1} \text { and }\left|\mu_{2}\right|<\lambda_{2}
\end{gathered}
$$

and

$$
\left|\mu_{1}\right|<\lambda_{1} \text { and }\left|\mu_{2}\right|>\lambda_{2}
$$

Theorem 3.2: Assume that (1.4), and (3.4) or (3.6) or [(1.3) and (3.5)] or [(1.3) and (3.7)] are satisfied, and let $\mathscr{U}_{0} \in \mathscr{H}$. Then there exists a positive constant $\mu_{j}^{0}$ independent of $\mu_{j}$, where $j=1$ in cases (3.4) and (3.6), and $j=2$ in cases (3.5) and (3.7), such that, if

$$
\mu_{j}^{2}+\left|\mu_{j}\right|<\mu_{j}^{0}
$$

the energy E satisfies (3.3).

Our last stability result concerns the case when (1.4) does not hold but (3.4) or (3.5) holds.

Theorem 3.3: Assume that (1.4) does not hold, and (3.4) or [(1.3) and (3.5)] hold, and let $\mathscr{U}_{0} \in$ $D(\mathscr{A})$. Then there exists a positive constant $\mu_{j}^{0}$ independent of $\mu_{j}$, where $j=1$ in case (3.4), and $j=2$ in case (3.5), such that, if (3.8) holds, then there exists a positive constant $c_{1}$ for which $E$ satisfies

$$
E(t) \leq \frac{c_{1}}{t}, \quad \forall t>0
$$

We will use $c$ (sometimes $c_{\delta}$ which depends on some parameter $\delta$ ), throughout the proof of our stability results, to denote a generic positive constant which depends continuously on the initial data and can be different from step to step, but it does not depend neither on $\lambda_{j}$ nor on $\mu_{j}$.

By considering suitable multipliers in the next lemmas, we will construct a Lyapunov functional $F$ satisfying some differential inequalities, for all $\mathscr{U}_{0} \in D(\mathscr{A})$; so all the calculations are justified. By integrating these differential inequalities, we get the desired decay estimates (3.3) and (3.9). In case of Theorem 3.1 and Theorem 3.2, by a simple density arguments $(D(\mathscr{A})$ is dense in $\mathscr{H}),(3.3)$ remains valid for any $\mathscr{U}_{0} \in \mathscr{H}$.

Before starting the proof of our stability results, we give the following identity:

Lemma 3.4: The energy functional satisfies

$$
E^{\prime}(t) \leq \int_{0}^{L}\left(d_{1} \varphi_{t}^{2}+d_{2} \psi_{t}^{2}\right) \mathrm{d} x
$$

where

$$
d_{j}= \begin{cases}\frac{-\lambda_{j}+\left|\mu_{j}\right|}{2} & \text { if } 0<\left|\mu_{j}\right| \leq \lambda_{j} \\ -\lambda_{j}+\left|\mu_{j}\right| & \text { if }\left|\mu_{j}\right|>\lambda_{j} \text { or } \mu_{j}=0\end{cases}
$$


Proof: By exploiting (2.3), (2.4), and (2.19), we obtain

$$
\begin{aligned}
E^{\prime}(t) \leq & \left(\frac{\xi_{1}}{2 \tau_{1}}+\frac{\left|\mu_{1}\right|}{2}-\lambda_{1}\right) \int_{0}^{L} \varphi_{t}^{2} \mathrm{~d} x+\left(\frac{\xi_{2}}{2 \tau_{2}}+\frac{\left|\mu_{2}\right|}{2}-\lambda_{2}\right) \int_{0}^{L} \psi_{t}^{2} \mathrm{~d} x \\
& +\left(\frac{\left|\mu_{1}\right|}{2}-\frac{\xi_{1}}{2 \tau_{1}}\right) \int_{0}^{L} \varphi_{t}^{2}\left(x, t-\tau_{1}\right) \mathrm{d} x+\left(\frac{\left|\mu_{2}\right|}{2}-\frac{\xi_{2}}{2 \tau_{2}}\right) \int_{0}^{L} \psi_{t}^{2}\left(x, t-\tau_{2}\right) \mathrm{d} x
\end{aligned}
$$

Then, using (2.11), we see that, if $0<\left|\mu_{j}\right| \leq \lambda_{j}$,

$$
\frac{\xi_{j}}{2 \tau_{j}}+\frac{\left|\mu_{j}\right|}{2}-\lambda_{j}=\frac{\left|\mu_{j}\right|}{2}-\frac{\xi_{j}}{2 \tau_{j}}=-\frac{\lambda_{j}-\left|\mu_{j}\right|}{2} \leq 0 .
$$

However, if $\left|\mu_{j}\right|>\lambda_{j}$ or $\mu_{j}=0$, we have

$$
\frac{\xi_{j}}{2 \tau_{j}}+\frac{\left|\mu_{j}\right|}{2}-\lambda_{j}=\left|\mu_{j}\right|-\lambda_{j} \quad \text { and } \quad \frac{\left|\mu_{j}\right|}{2}-\frac{\xi_{j}}{2 \tau_{j}}=0 .
$$

Hence, (3.12) yields (3.10).

Remark 3.5: When (3.2) or (3.4) or [(1.3) and (3.5)] hold, $E^{\prime} \leq 0$, and then (1.1) is dissipative. However, when (3.6) or [(1.3) and (3.7)] hold, the sign of $E^{\prime}$ is not determined from (3.10), and therefore, (1.1) is not necessarily dissipative with respect to $E$ at this stage.

\section{Proof of Theorem 3.1}

Assume that (3.2) holds and let $\mathscr{U}_{0} \in D(\mathscr{A})$.

Lemma 4.1: The functional

$$
J(t):=\int_{0}^{L}\left(\rho_{1} \varphi \varphi_{t}+\rho_{2} \psi \psi_{t}+\frac{\lambda_{1}}{2} \varphi^{2}+\frac{\lambda_{2}}{2} \psi^{2}\right) \mathrm{d} x
$$

satisfies, for any $\delta>0$,

$$
\begin{aligned}
J^{\prime}(t) \leq & -k_{1} \int_{0}^{L}\left(\varphi_{x}+\psi\right)^{2} \mathrm{~d} x-k_{2} \int_{0}^{L} \psi_{x}^{2} \mathrm{~d} x+\int_{0}^{L}\left(\rho_{1} \varphi_{t}^{2}+\rho_{2} \psi_{t}^{2}\right) \mathrm{d} x \\
& +\delta \int_{0}^{L}\left(\varphi_{x}^{2}+\psi_{x}^{2}\right) \mathrm{d} x+c_{\delta} \int_{0}^{L}\left(\mu_{1}^{2} \varphi_{t}^{2}\left(x, t-\tau_{1}\right)+\mu_{2}^{2} \psi_{t}^{2}\left(x, t-\tau_{2}\right)\right) \mathrm{d} x
\end{aligned}
$$

Proof: By differentiating $J$, and using the first two equations in (1.1) and boundary conditions (1.2) or (1.3), we have

$$
\begin{aligned}
J^{\prime}(t)= & \int_{0}^{L}\left(\rho_{1} \varphi_{t}^{2}+\rho_{2} \psi_{t}^{2}\right) \mathrm{d} x-\int_{0}^{L}\left(k_{1}\left(\varphi_{x}+\psi\right)^{2}+k_{2} \psi_{x}^{2}\right) \mathrm{d} x \\
& -\int_{0}^{L}\left(\mu_{1} \varphi \varphi_{t}\left(x, t-\tau_{1}\right)+\mu_{2} \psi \psi_{t}\left(x, t-\tau_{2}\right)\right) \mathrm{d} x
\end{aligned}
$$

Consequently, applying Young's inequality, for the terms of the last integral of the above equality, and using Poincarés inequality (2.12), for $\varphi$ and $\psi$, we find (4.2). 
Lemma 4.2: The functionals

$$
\left\{\begin{array}{l}
I_{1}(t)=\xi_{1} \int_{0}^{L} \int_{0}^{1} e^{-2 \tau_{1} \rho} \varphi_{t}^{2}\left(x, t-\tau_{1} \rho\right) \mathrm{d} \rho \mathrm{d} x \\
I_{2}(t)=\xi_{2} \int_{0}^{L} \int_{0}^{1} e^{-2 \tau_{2} \rho} \psi_{t}^{2}\left(x, t-\tau_{2} \rho\right) \mathrm{d} \rho \mathrm{d} x
\end{array}\right.
$$

satisfy

$$
\begin{aligned}
I_{1}^{\prime}(t) \leq & -2 \xi_{1} e^{-2 \tau_{1}} \int_{0}^{L} \int_{0}^{1} \varphi_{t}^{2}\left(x, t-\tau_{1} \rho\right) \mathrm{d} \rho \mathrm{d} x+\frac{\xi_{1}}{\tau_{1}} \int_{0}^{L} \varphi_{t}^{2} \mathrm{~d} x \\
& -\frac{\xi_{1} e^{-2 \tau_{1}}}{\tau_{1}} \int_{0}^{L} \varphi_{t}^{2}\left(x, t-\tau_{1}\right) \mathrm{d} x
\end{aligned}
$$

and

$$
\begin{aligned}
I_{2}^{\prime}(t) \leq & -2 \xi_{2} e^{-2 \tau_{2}} \int_{0}^{L} \int_{0}^{1} \psi_{t}^{2}\left(x, t-\tau_{2} \rho\right) \mathrm{d} \rho \mathrm{d} x+\frac{\xi_{2}}{\tau_{2}} \int_{0}^{L} \psi_{t}^{2} \mathrm{~d} x \\
& -\frac{\xi_{2} e^{-2 \tau_{2}}}{\tau_{2}} \int_{0}^{L} \psi_{t}^{2}\left(x, t-\tau_{2}\right) \mathrm{d} x
\end{aligned}
$$

Proof: Using (2.1) and (2.2), the derivative of $I_{1}$ entails

$$
\begin{aligned}
I_{1}^{\prime}(t) & =2 \xi_{1} \int_{0}^{L} \int_{0}^{1} e^{-2 \tau_{1} \rho} \varphi_{t t}\left(x, t-\tau_{1} \rho\right) \varphi_{t}\left(x, t-\tau_{1} \rho\right) \mathrm{d} \rho \mathrm{d} x \\
& =-\frac{2 \xi_{1}}{\tau_{1}} \int_{0}^{L} \int_{0}^{1} e^{-2 \tau_{1} \rho} \varphi_{t \rho}\left(x, t-\tau_{1} \rho\right) \varphi_{t}\left(x, t-\tau_{1} \rho\right) \mathrm{d} \rho \mathrm{d} x \\
& =-\frac{\xi_{1}}{\tau_{1}} \int_{0}^{L} \int_{0}^{1} e^{-2 \tau_{1} \rho} \partial_{\rho} \varphi_{t}^{2}\left(x, t-\tau_{1} \rho\right) \mathrm{d} \rho \mathrm{d} x
\end{aligned}
$$

Then, using an integrating by parts, the above formula can be rewritten as

$$
I_{1}^{\prime}(t)=-2 \xi_{1} \int_{0}^{L} \int_{0}^{1} e^{-2 \tau_{1} \rho} \varphi_{t}^{2}\left(x, t-\tau_{1} \rho\right) \mathrm{d} \rho \mathrm{d} x+\frac{\xi_{1}}{\tau_{1}} \int_{0}^{L} \varphi_{t}^{2} \mathrm{~d} x-\frac{\xi_{1} e^{-2 \tau_{1}}}{\tau_{1}} \int_{0}^{L} \varphi_{t}^{2}\left(x, t-\tau_{1}\right) \mathrm{d} x
$$

which gives (4.4), since $-e^{-2 \tau_{1} \rho} \leq-e^{-2 \tau_{1}}$, for any $\left.\rho \in\right] 0,1$ [. Similarily, (4.6) can be proved.

Now, let $N_{1}, N_{2}>0$ and

$$
F=N_{1} E+N_{2}\left(I_{1}+I_{2}\right)+J .
$$


By combining (4.2), (4.4), and (4.6), we obtain

$$
\begin{aligned}
F^{\prime}(t) \leq & N_{1} E^{\prime}(t)-k_{1} \int_{0}^{L}\left(\varphi_{x}+\psi\right)^{2} \mathrm{~d} x-k_{2} \int_{0}^{L} \psi_{x}^{2} \mathrm{~d} x+\delta \int_{0}^{L}\left(\varphi_{x}^{2}+\psi_{x}^{2}\right) \mathrm{d} x \\
& +\int_{0}^{L}\left(\left(\rho_{1}+\frac{\xi_{1} N_{2}}{\tau_{1}}\right) \varphi_{t}^{2}+\left(\rho_{2}+\frac{\xi_{2} N_{2}}{\tau_{2}}\right) \psi_{t}^{2}\right) \mathrm{d} x \\
& +\left(c_{\delta} \mu_{1}^{2}-\frac{\xi_{1} N_{2} e^{-2 \tau_{1}}}{\tau_{1}}\right) \int_{0}^{L} \varphi_{t}^{2}\left(x, t-\tau_{1}\right) \mathrm{d} x \\
& +\left(c_{\delta} \mu_{2}^{2}-\frac{\xi_{2} N_{2} e^{-2 \tau_{2}}}{\tau_{2}}\right) \int_{0}^{L} \psi_{t}^{2}\left(x, t-\tau_{2}\right) \mathrm{d} x \\
& -2 N_{2} \int_{0}^{L} \int_{0}^{1}\left(\xi_{1} e^{-2 \tau_{1}} \varphi_{t}^{2}\left(x, t-\tau_{1} \rho\right)+\xi_{2} e^{-2 \tau_{2}} \psi_{t}^{2}\left(x, t-\tau_{2} \rho\right)\right) \mathrm{d} \rho \mathrm{d} x .
\end{aligned}
$$

At this point, we choose $\delta>0$ small enough and $N_{2}>0$ large enough so that ( $c_{0}$ is defined in (2.12))

$$
\delta \max \left\{\frac{2}{k_{1}}, \frac{2 c_{0}+1}{k_{2}}\right\} \leq \frac{1}{2} \quad \text { and } \min \left\{\xi_{1}, \xi_{2}\right\} N_{2} \geq c_{\delta} \max \left\{\tau_{1} e^{2 \tau_{1}} \mu_{1}^{2}, \tau_{2} e^{2 \tau_{2}} \mu_{2}^{2}\right\}
$$

(notice that, thanks to (2.11), $\xi_{j}=0$ implies $\mu_{j}=0$; so $N_{2}$ exists). Therefore, using (2.13), for $(\varphi, \psi)$ instead of $\left(u_{1}, v_{1}\right)$, and the definition of $E$, we find that (4.8) implies that

$$
F^{\prime}(t) \leq N_{1} E^{\prime}(t)-c E(t)+\tilde{c} \int_{0}^{L}\left(\varphi_{t}^{2}+\psi_{t}^{2}\right) \mathrm{d} x,
$$

for some positive constant $\tilde{c}$ depending on $\lambda_{j}$ and $\mu_{j}$. Hence, according to (3.2) and (3.10), we have (notice that $\max \left\{d_{1}, d_{2}\right\}<0$ )

$$
\int_{0}^{L}\left(\varphi_{t}^{2}+\psi_{t}^{2}\right) \mathrm{d} x \leq \frac{1}{\max \left\{d_{1}, d_{2}\right\}} E^{\prime}(t) .
$$

By combining the above two inequalities, we get

$$
F^{\prime}(t) \leq\left(N_{1}+\frac{\tilde{c}}{\max \left\{d_{1}, d_{2}\right\}}\right) E^{\prime}(t)-c E(t) .
$$

On the other hand, using again (2.13) (for $(\varphi, \psi)$ instead of $\left.\left(u_{1}, v_{1}\right)\right)$ and the definition of $J, I_{1}$ and $I_{2}$, we can find that there exists a positive constant $\beta$ (not depending on $N_{1}$ ) such that

$$
\left|N_{2}\left(I_{1}+I_{2}\right)+J\right| \leq \beta E,
$$

which implies that

$$
\left(N_{1}-\beta\right) E \leq F \leq\left(N_{1}+\beta\right) E .
$$

Thus, choosing $N_{1}$ large enough so that

$$
N_{1}+\frac{\tilde{c}}{\max \left\{d_{1}, d_{2}\right\}} \geq 0 \quad \text { and } \quad N_{1}>\beta,
$$


and using the fact that $E^{\prime} \leq 0$ (see Remark (3.5)), we deduce from (4.9) and (4.10) that, for some positive constant $c_{1}$,

$$
F^{\prime} \leq-c_{1} F
$$

Then, by integrating (4.11) over [0,t] and using again (4.10), we get (3.3).

\section{Proof of Theorem 3.2}

Assume that (1.4) is satisfied, (3.4) or (3.5) or (3.6) or (3.7) holds and let $\mathscr{U}_{0} \in D(\mathscr{A})$. We distinguish two cases.

Case 1: the boundary conditions (1.2) or (1.3) hold,

$$
\left|\mu_{1}\right| \geq \lambda_{1} \quad \text { and } \quad\left|\mu_{2}\right|<\lambda_{2}
$$

Lemma 5.1: The functional

$$
I(t)=-\int_{0}^{L}\left(\rho_{1} \varphi \varphi_{t}+\rho_{2} \psi \psi_{t}+\frac{\lambda_{1}}{2} \varphi^{2}+\frac{\lambda_{2}}{2} \psi^{2}\right) \mathrm{d} x
$$

satisfies

$$
\begin{aligned}
I^{\prime}(t) \leq & -\int_{0}^{L}\left(\rho_{1} \varphi_{t}^{2}+\rho_{2} \psi_{t}^{2}\right) \mathrm{d} x+\int_{0}^{L}\left(k_{1}\left(\varphi_{x}+\psi\right)^{2}+k_{2} \psi_{x}^{2}\right) \mathrm{d} x \\
& +\delta \int_{0}^{L}\left(\varphi_{x}^{2}+\psi_{x}^{2}\right) \mathrm{d} x+c_{\delta} \int_{0}^{L}\left(\mu_{1}^{2} \varphi_{t}^{2}\left(x, t-\tau_{1}\right)+\mu_{2}^{2} \psi_{t}^{2}\left(x, t-\tau_{2}\right)\right) \mathrm{d} x .
\end{aligned}
$$

Proof: The proof of (5.2) is identical to the one of (4.2).

Similarly to [3], we consider the following lemma:

Lemma 5.2: The functional

$$
I_{3}(t)=\rho_{2} \int_{0}^{L} \psi_{t}\left(\varphi_{x}+\psi\right) \mathrm{d} x+\frac{k_{2} \rho_{1}}{k_{1}} \int_{0}^{L} \psi_{x} \varphi_{t} \mathrm{~d} x
$$

satisfies, for any $\epsilon, \delta>0$,

$$
\begin{aligned}
I_{3}^{\prime}(t) \leq & \frac{k_{2}^{2}}{2 \epsilon}\left(\psi_{x}^{2}(L, t)+\psi_{x}^{2}(0, t)\right)+\frac{\epsilon}{2}\left(\varphi_{x}^{2}(L, t)+\varphi_{x}^{2}(0, t)\right)+\rho_{2} \int_{0}^{L} \psi_{t}^{2} \mathrm{~d} x \\
& -\left(k_{1}-\delta\right) \int_{0}^{L}\left(\varphi_{x}+\psi\right)^{2} \mathrm{~d} x+\delta \int_{0}^{L} \psi_{x}^{2} \mathrm{~d} x+\left(\frac{k_{2} \rho_{1}}{k_{1}}-\rho_{2}\right) \int_{0}^{L} \varphi_{t} \psi_{x t} \mathrm{~d} x \\
& +c_{\delta} \int_{0}^{L}\left(\lambda_{1}^{2} \varphi_{t}^{2}+\lambda_{2}^{2} \psi_{t}^{2}+\mu_{1}^{2} \varphi_{t}^{2}\left(x, t-\tau_{1}\right)+\mu_{2}^{2} \psi_{t}^{2}\left(x, t-\tau_{2}\right)\right) \mathrm{d} x
\end{aligned}
$$


in case (1.2), and

$$
\begin{aligned}
I_{3}^{\prime}(t) \leq & \rho_{2} \int_{0}^{L} \psi_{t}^{2} \mathrm{~d} x-\left(k_{1}-\delta\right) \int_{0}^{L}\left(\varphi_{x}+\psi\right)^{2} \mathrm{~d} x \\
& +\delta \int_{0}^{L} \psi_{x}^{2} \mathrm{~d} x+\left(\frac{k_{2} \rho_{1}}{k_{1}}-\rho_{2}\right) \int_{0}^{L} \varphi_{t} \psi_{x t} \mathrm{~d} x \\
& +c_{\delta} \int_{0}^{L}\left(\lambda_{1}^{2} \varphi_{t}^{2}+\lambda_{2}^{2} \psi_{t}^{2}+\mu_{1}^{2} \varphi_{t}^{2}\left(x, t-\tau_{1}\right)+\mu_{2}^{2} \psi_{t}^{2}\left(x, t-\tau_{2}\right)\right) \mathrm{d} x
\end{aligned}
$$

in case (1.3).

Proof: Using equations in (1.1) and the boundary conditions (1.2), and arguing as before, we have

$$
\begin{aligned}
I_{3}^{\prime}(t)= & -k_{1} \int_{0}^{L}\left(\varphi_{x}+\psi\right)^{2} \mathrm{~d} x+\rho_{2} \int_{0}^{L} \psi_{t}^{2} \mathrm{~d} x+\left(\frac{k_{2} \rho_{1}}{k_{1}}-\rho_{2}\right) \int_{0}^{L} \varphi_{t} \psi_{x t} \mathrm{~d} x \\
& +k_{2}\left(\varphi_{x}(L, t) \psi_{x}(L, t)-\varphi_{x}(0, t) \psi_{x}(0, t)\right)-\int_{0}^{L}\left(\varphi_{x}+\psi\right)\left(\lambda_{2} \psi_{t}+\mu_{2} \psi_{t}\left(x, t-\tau_{2}\right)\right) \mathrm{d} x \\
& -\frac{k_{2}}{k_{1}} \int_{0}^{L} \psi_{x}\left(\lambda_{1} \varphi_{t}+\mu_{1} \varphi_{t}\left(x, t-\tau_{1}\right)\right) \mathrm{d} x .
\end{aligned}
$$

Using Young's inequality (for the last three terms of this equality), (5.3) is established. Similarly, in case (1.3), we get

$$
\begin{aligned}
I_{3}^{\prime}(t)= & -k_{1} \int_{0}^{L}\left(\varphi_{x}+\psi\right)^{2} \mathrm{~d} x+\rho_{2} \int_{0}^{L} \psi_{t}^{2} \mathrm{~d} x+\left(\frac{k_{2} \rho_{1}}{k_{1}}-\rho_{2}\right) \int_{0}^{L} \varphi_{t} \psi_{x t} \mathrm{~d} x \\
& -\int_{0}^{L}\left(\varphi_{x}+\psi\right)\left(\lambda_{2} \psi_{t}+\mu_{2} \psi_{t}\left(x, t-\tau_{2}\right)\right) \mathrm{d} x-\frac{k_{2}}{k_{1}} \int_{0}^{L} \psi_{x}\left(\lambda_{1} \varphi_{t}+\mu_{1} \varphi_{t}\left(x, t-\tau_{1}\right)\right) \mathrm{d} x,
\end{aligned}
$$

which, using Young's inequality (for the last two terms of this equality), implies (5.27).

To estimate the boundary terms in (5.3), we proceed as in [3].

Lemma 5.3: Let $m(x)=2-\frac{4}{L} x$. Then, for any $\epsilon>0$, the functionals

$$
I_{4}=\rho_{2} k_{2} \int_{0}^{L} m(x) \psi_{t} \psi_{x} \mathrm{~d} x \text { and } I_{5}=\rho_{1} \int_{0}^{L} m(x) \varphi_{t} \varphi_{x} \mathrm{~d} x
$$

satisfy

$$
\begin{aligned}
I_{4}^{\prime}(t) \leq & -k_{2}^{2}\left(\psi_{x}^{2}(L, t)+\psi_{x}^{2}(0, t)\right)+\epsilon k_{1} \int_{0}^{L}\left(\varphi_{x}+\psi\right)^{2} \mathrm{~d} x+c \int_{0}^{L} \psi_{t}^{2} \mathrm{~d} x \\
& +c\left(1+\frac{1}{\varepsilon}\right) \int_{0}^{L} \psi_{x}^{2} \mathrm{~d} x+c \int_{0}^{L}\left(\lambda_{2}^{2} \psi_{t}^{2}+\mu_{2}^{2} \psi_{t}^{2}\left(x, t-\tau_{2}\right)\right) \mathrm{d} x
\end{aligned}
$$

and

$$
\begin{aligned}
I_{5}^{\prime}(t) \leq & -k_{1}\left(\varphi_{x}^{2}(L, t)+\varphi_{x}^{2}(0, t)\right)+c \int_{0}^{L}\left(\varphi_{t}^{2}+\varphi_{x}^{2}+\psi_{x}^{2}\right) \mathrm{d} x \\
& +c \int_{0}^{L}\left(\lambda_{1}^{2} \varphi_{t}^{2}+\mu_{1}^{2} \varphi_{t}^{2}\left(x, t-\tau_{1}\right)\right) \mathrm{d} x .
\end{aligned}
$$


Proof: Exploiting the second equation in (1.1) and using the boundary conditions (1.2), we get

$$
\begin{aligned}
I_{4}^{\prime}(t)= & \frac{2 \rho_{2} k_{2}}{L} \int_{0}^{L} \psi_{t}^{2} \mathrm{~d} x+\frac{2 k_{2}^{2}}{L} \int_{0}^{L} \psi_{x}^{2} \mathrm{~d} x-k_{2}^{2}\left(\psi_{x}^{2}(L, t)+\psi_{x}^{2}(0, t)\right) \\
& -k_{2} \int_{0}^{L} m \psi_{x}\left(k_{1}\left(\varphi_{x}+\psi\right)+\lambda_{2} \psi_{t}+\mu_{2} \psi_{t}\left(x, t-\tau_{2}\right)\right) \mathrm{d} x
\end{aligned}
$$

Using Young's inequality, for the terms of the last integral of the above equality, (5.5) is established. Similarly, exploiting the first equation in (1.1) and using the boundary conditions (1.2), we find

$$
\begin{aligned}
I_{5}^{\prime}(t)= & \frac{2 \rho_{1}}{L} \int_{0}^{L} \varphi_{t}^{2} \mathrm{~d} x+\frac{2 k_{1}}{L} \int_{0}^{L} \varphi_{x}^{2} \mathrm{~d} x-k_{1}\left(\varphi_{x}^{2}(L, t)+\varphi_{x}^{2}(0, t)\right) \\
& +k_{1} \int_{0}^{L} m \varphi_{x} \psi_{x} \mathrm{~d} x-\int_{0}^{L} m \varphi_{x}\left(\lambda_{1} \varphi_{t}+\mu_{1} \varphi_{t}\left(x, t-\tau_{1}\right)\right) \mathrm{d} x .
\end{aligned}
$$

Then (5.6) can be proved by applying Young's inequality, for the terms of the last two integrals of the above equality.

Lemma 5.4: For any $\epsilon \in] 0,1[$ and $\delta>0$, the functional

$$
I_{6}= \begin{cases}I_{3}+\frac{1}{2 \epsilon} I_{4}+\frac{\epsilon}{2 k_{1}} I_{5}, & \text { in case }(1.2) \\ I_{3}, & \text { in case }(1.3)\end{cases}
$$

satisfies

$$
\begin{aligned}
I_{6}^{\prime}(t) \leq & -\left(\frac{k_{1}}{2}-\delta-c \epsilon\right) \int_{0}^{L}\left(\varphi_{x}+\psi\right)^{2} \mathrm{~d} x+\frac{c}{\epsilon} \int_{0}^{L} \psi_{t}^{2} \mathrm{~d} x \\
& +\left(\delta+\frac{c}{\epsilon^{2}}\right) \int_{0}^{L} \psi_{x}^{2} \mathrm{~d} x+c \epsilon \int_{0}^{L} \varphi_{t}^{2} \mathrm{~d} x+\left(\frac{\rho_{1} k_{2}}{k_{1}}-\rho_{2}\right) \int_{0}^{L} \varphi_{t} \psi_{x t} \mathrm{~d} x \\
& +\left(c_{\epsilon}+c_{\delta}\right) \int_{0}^{L}\left(\lambda_{1}^{2} \varphi_{t}^{2}+\lambda_{2}^{2} \psi_{t}^{2}+\mu_{1}^{2} \varphi_{t}^{2}\left(x, t-\tau_{1}\right)+\mu_{2}^{2} \psi_{t}^{2}\left(x, t-\tau_{2}\right)\right) \mathrm{d} x .
\end{aligned}
$$

Proof: Using Poincaré's inequality (2.12), for $\psi$, we obtain

$$
\begin{aligned}
\int_{0}^{L} \varphi_{x}^{2} \mathrm{~d} x & \leq 2 \int_{0}^{L}\left(\varphi_{x}+\psi\right)^{2} \mathrm{~d} x+2 \int_{0}^{L} \psi^{2} \mathrm{~d} x \\
& \leq 2 \int_{0}^{L}\left(\varphi_{x}+\psi\right)^{2} \mathrm{~d} x+c \int_{0}^{L} \psi_{x}^{2} \mathrm{~d} x
\end{aligned}
$$

Then (5.3)-(5.6) imply (5.8).

Lemma 5.5: The functional $I_{7}=I_{6}+\frac{1}{8}$ I satisfies

$$
\begin{aligned}
I_{7}^{\prime}(t) \leq & -\frac{k_{1}}{4} \int_{0}^{L}\left(\varphi_{x}+\psi\right)^{2} \mathrm{~d} x-\frac{\rho_{1}}{16} \int_{0}^{L} \varphi_{t}^{2} \mathrm{~d} x+c \int_{0}^{L}\left(\psi_{t}^{2}+\psi_{x}^{2}\right) \mathrm{d} x \\
& +\delta c \int_{0}^{L}\left(\left(\varphi_{x}+\psi\right)^{2}+\psi_{x}^{2}\right) \mathrm{d} x+\left(\frac{\rho_{1} k_{2}}{k_{1}}-\rho_{2}\right) \int_{0}^{L} \varphi_{t} \psi_{x t} \mathrm{~d} x \\
& +c_{\delta} \int_{0}^{L}\left(\lambda_{1}^{2} \varphi_{t}^{2}+\lambda_{2}^{2} \psi_{t}^{2}+\mu_{1}^{2} \varphi_{t}^{2}\left(x, t-\tau_{1}\right)+\mu_{2}^{2} \psi_{t}^{2}\left(x, t-\tau_{2}\right)\right) \mathrm{d} x .
\end{aligned}
$$

Proof: Using (5.9), inequalities (5.2) and (5.8) (with $\epsilon \in] 0,1$ [ small enough) imply (5.10). 
Now, as in [3], we use a function $w$ to get a crucial estimate.

Lemma 5.6: The function

$$
w(x, t)=-\int_{0}^{x} \psi(y, t) \mathrm{d} y+\frac{1}{L}\left(\int_{0}^{L} \psi(y, t) \mathrm{d} y\right) x
$$

satisfies the estimates

$$
\int_{0}^{L} w_{x}^{2} \mathrm{~d} x \leq c \int_{0}^{L} \psi^{2} \mathrm{~d} x, \quad \forall t \geq 0
$$

and

$$
\int_{0}^{L} w_{t}^{2} \mathrm{~d} x \leq c \int_{0}^{L} \psi_{t}^{2} \mathrm{~d} x, \quad \forall t \geq 0 .
$$

Proof: We just have to calculate $w_{x}$ and use Hölder's inequality to get (5.12). Applying (5.12) to $w_{t}$, we get

$$
\int_{0}^{L} w_{x t}^{2} \mathrm{~d} x \leq c \int_{0}^{L} \psi_{t}^{2} \mathrm{~d} x, \quad \forall t \geq 0
$$

Then, using Poincaré's inequality (2.12), for $w_{t}$ (note that $w_{t}(0, t)=w_{t}(L, t)=0$ ), we arrive at (5.13).

Lemma 5.7: For any $\epsilon \in] 0,1[$ and $\delta>0$, the functional

$$
I_{8}(t)=\int_{0}^{L}\left(\rho_{2} \psi \psi_{t}+\rho_{1} w \varphi_{t}+\frac{\lambda_{2}}{2} \psi^{2}\right) \mathrm{d} x
$$

satisfies

$$
\begin{aligned}
I_{8}^{\prime}(t) \leq & -k_{2} \int_{0}^{L} \psi_{x}^{2} \mathrm{~d} x+\frac{c}{\epsilon} \int_{0}^{L} \psi_{t}^{2} \mathrm{~d} x+\epsilon \int_{0}^{L} \varphi_{t}^{2} \mathrm{~d} x+\delta \int_{0}^{L} \psi_{x}^{2} \mathrm{~d} x \\
& +c_{\delta} \int_{0}^{L}\left(\lambda_{1}^{2} \varphi_{t}^{2}+\mu_{1}^{2} \varphi_{t}^{2}\left(x, t-\tau_{1}\right)+\mu_{2}^{2} \psi_{t}^{2}\left(x, t-\tau_{2}\right)\right) \mathrm{d} x .
\end{aligned}
$$

Proof: Exploiting the first two equations in (1.1), integrating by parts and using the boundary conditions (1.2) or (1.3), we get

$$
\begin{aligned}
I_{8}^{\prime}(t)= & \int_{0}^{L}\left(\rho_{2} \psi_{t}^{2}-k_{2} \psi_{x}^{2}\right) \mathrm{d} x-k_{1} \int_{0}^{L}\left(\varphi_{x}+\psi\right)\left(\psi+w_{x}\right) \mathrm{d} x \\
& +\rho_{1} \int_{0}^{L} w_{t} \varphi_{t} \mathrm{~d} x-\mu_{2} \int_{0}^{L} \psi \psi_{t}\left(x, t-\tau_{2}\right) \mathrm{d} x \\
& -\int_{0}^{L} w\left(\lambda_{1} \varphi_{t}+\mu_{1} \varphi_{t}\left(x, t-\tau_{1}\right)\right) \mathrm{d} x .
\end{aligned}
$$


Notice that

$$
\begin{aligned}
-k_{1} \int_{0}^{L}\left(\varphi_{x}+\psi\right)\left(\psi+w_{x}\right) \mathrm{d} x & =-k_{1} \int_{0}^{L}\left(\varphi_{x}+\psi\right)\left(\frac{1}{L} \int_{0}^{L} \psi(y, t) \mathrm{d} y\right) \mathrm{d} x \\
& =-\frac{k_{1}}{L}\left(\int_{0}^{L} \psi(y, t) \mathrm{d} y\right)^{2} \\
& \leq 0
\end{aligned}
$$

Then, by applying Young's inequality, for the terms of the last three integrals of (5.29), and using (5.12) and (5.13), (5.14) is established.

Now, for $N_{1}, N_{2}, N_{3}>0$, let

$$
F=N_{1} E+N_{2}\left(I_{1}+I_{2}\right)+N_{3} I_{8}+I_{7}
$$

By combining (4.4), (4.6), (5.10) and (5.14), we obtain

$$
\begin{aligned}
F^{\prime}(t) \leq & -\left(k_{2} N_{3}-c-\delta\left(N_{3}+c\right)\right) \int_{0}^{L} \psi_{x}^{2} \mathrm{~d} x-\left(\frac{\rho_{1}}{16}-\epsilon N_{3}\right) \int_{0}^{L} \varphi_{t}^{2} \mathrm{~d} x \\
& +N_{1} E^{\prime}(t)+\left(\frac{c N_{3}}{\epsilon}+c+\frac{\xi_{2} N_{2}}{\tau_{2}}\right) \int_{0}^{L} \psi_{t}^{2} \mathrm{~d} x-\left(\frac{k_{1}}{4}-\delta c\right) \int_{0}^{L}\left(\varphi_{x}+\psi\right)^{2} \mathrm{~d} x \\
& -2 N_{2} \int_{0}^{L} \int_{0}^{1}\left(\xi_{1} e^{-2 \tau_{1}} \varphi_{t}^{2}\left(x, t-\tau_{1} p\right)+\xi_{2} e^{-2 \tau_{2}} \psi_{t}^{2}\left(x, t-\tau_{2} p\right)\right) \mathrm{d} p \mathrm{~d} x \\
& +\frac{\xi_{1} N_{2}}{\tau_{1}} \int_{0}^{L} \varphi_{t}^{2} \mathrm{~d} x+\left(c_{\delta} \mu_{1}^{2}\left(N_{3}+1\right)-\frac{\xi_{1} e^{-2 \tau_{1}}}{\tau_{1}} N_{2}\right) \int_{0}^{L} \varphi_{t}^{2}\left(x, t-\tau_{1}\right) \mathrm{d} x \\
& +\left(c_{\delta} \mu_{2}^{2}\left(N_{3}+1\right)-\frac{\xi_{2} e^{-2 \tau_{2}}}{\tau_{2}} N_{2}\right) \int_{0}^{L} \psi_{t}^{2}\left(x, t-\tau_{2}\right) \mathrm{d} x \\
& +c_{\delta} \int_{0}^{L}\left(\lambda_{1}^{2}\left(N_{3}+1\right) \varphi_{t}^{2}+\lambda_{2}^{2} \psi_{t}^{2}\right) \mathrm{d} x+\left(\frac{\rho_{1} k_{2}}{k_{1}}-\rho_{2}\right) \int_{0}^{L} \varphi_{t} \psi_{x t} \mathrm{~d} x .
\end{aligned}
$$

At this point, we choose $N_{3}>0$ large enough so that

$$
k_{2} N_{3}-c>0
$$

then $\epsilon \in] 0,1[$ and $\delta>0$ small enough so that

$$
\frac{\rho_{1}}{16}-\epsilon N_{3}>0, \quad \delta\left(N_{3}+c\right)<k_{2} N_{3}-c \quad \text { and } \delta c<\frac{k_{1}}{4} .
$$

Next, we pick $N_{2}>0$ large enough so that

$$
c_{\delta} \mu_{1}^{2}\left(N_{3}+1\right)-\frac{\xi_{1} e^{-2 \tau_{1}}}{\tau_{1}} N_{2} \leq 0 \quad \text { and } \quad c_{\delta} \mu_{2}^{2}\left(N_{3}+1\right)-\frac{\xi_{2} e^{-2 \tau_{2}}}{\tau_{2}} N_{2} \leq 0
$$

Notice that, according to (2.11), if $\mu_{j}=0$, then $\xi_{j}=0$. Otherwise, in virtue of (2.11) and (5.1), $\xi_{1}=\tau_{1}\left|\mu_{1}\right|$ and $\xi_{2}=\tau_{2} \lambda_{2}$. So $N_{2}$ exists and can be taken in the form

$$
N_{2}=c\left(\left|\mu_{1}\right|+\lambda_{2}\right)
$$


From the choice (5.18), the definition of $E$ and the fact that $\lambda_{1} \leq\left|\mu_{1}\right|$, we deduce from (5.17) that

$$
\begin{aligned}
F^{\prime}(t) \leq & -c \min \left\{1,\left|\mu_{1}\right|+\lambda_{2}\right\} E(t)+N_{1} E^{\prime}(t)+c\left(\mu_{1}^{2}+\lambda_{2}\left|\mu_{1}\right|\right) \int_{0}^{L} \varphi_{t}^{2} \mathrm{~d} x \\
& +c\left(\lambda_{2}^{2}+\lambda_{2}\left|\mu_{1}\right|+1\right) \int_{0}^{L} \psi_{t}^{2} \mathrm{~d} x+\left(\frac{\rho_{1} k_{2}}{k_{1}}-\rho_{2}\right) \int_{0}^{L} \varphi_{t} \psi_{x t} \mathrm{~d} x
\end{aligned}
$$

Hence, according to (3.10) and (5.1), we have

$$
E^{\prime}(t) \leq \int_{0}^{L}\left(\left(\left|\mu_{1}\right|-\lambda_{1}\right) \varphi_{t}^{2}+\frac{\left|\mu_{2}\right|-\lambda_{2}}{2} \psi_{t}^{2}\right) \mathrm{d} x
$$

By combining (5.31) and (5.35), we get $\left(\min \left\{1,\left|\mu_{1}\right|+\lambda_{2}\right\} \geq \min \left\{1, \lambda_{2}\right\}\right)$

$$
\begin{aligned}
F^{\prime}(t) \leq & -c \min \left\{1, \lambda_{2}\right\} E(t)+\left(\frac{\rho_{1} k_{2}}{k_{1}}-\rho_{2}\right) \int_{0}^{L} \varphi_{t} \psi_{x t} \mathrm{~d} x \\
& +\left(c\left(\mu_{1}^{2}+\lambda_{2}\left|\mu_{1}\right|\right)+N_{1}\left(\left|\mu_{1}\right|-\lambda_{1}\right)\right) \int_{0}^{L} \varphi_{t}^{2} \mathrm{~d} x \\
& +\left(c\left(\lambda_{2}^{2}+\lambda_{2}\left|\mu_{1}\right|+1\right)+\frac{N_{1}}{2}\left(\left|\mu_{2}\right|-\lambda_{2}\right)\right) \int_{0}^{L} \psi_{t}^{2} \mathrm{~d} x .
\end{aligned}
$$

On the other hand, by definition of the functionals $I, I_{1}-I_{8}$ and $E$ (notice that $\lambda_{1} \leq\left|\mu_{1}\right|$ ), we have

$$
\left|N_{2}\left(I_{1}+I_{2}\right)+N_{3} I_{8}+I_{7}\right| \leq c\left(\left|\mu_{1}\right|+\lambda_{2}+1\right) E,
$$

which implies that

$$
\left(N_{1}-c\left(\left|\mu_{1}\right|+\lambda_{2}+1\right)\right) E \leq F \leq\left(N_{1}+c\left(\left|\mu_{1}\right|+\lambda_{2}+1\right)\right) E .
$$

Then we choose $N_{1}$ large enough such that

$$
c\left(\lambda_{2}^{2}+\lambda_{2}\left|\mu_{1}\right|+1\right)+\frac{N_{1}}{2}\left(\left|\mu_{2}\right|-\lambda_{2}\right) \leq 0 \quad \text { and } \quad N_{1}>c\left(\left|\mu_{1}\right|+\lambda_{2}+1\right) .
$$

Because $\left|\mu_{2}\right|<\lambda_{2}$, then $N_{1}$ exists and can be taken in the form

$$
N_{1}=c\left(\frac{\lambda_{2}^{2}+\lambda_{2}\left|\mu_{1}\right|+1}{\lambda_{2}-\left|\mu_{2}\right|}+\left|\mu_{1}\right|+\lambda_{2}+1\right),
$$

so the last term in (5.31) is non-positive and $F \sim E$. In addition, using the definition of $E$, we deduce from (5.31) and (5.22) that

$$
F^{\prime}(t) \leq \quad-c \min \left\{1, \lambda_{2}\right\} E(t)+\tilde{c}\left(\mu_{1}^{2}+\left|\mu_{1}\right|\right) E(t)+\left(\frac{\rho_{1} k_{2}}{k_{1}}-\rho_{2}\right) \int_{0}^{L} \varphi_{t} \psi_{x t} \mathrm{~d} x,
$$

where $\tilde{c}$ is a positive constant which depends on $\lambda_{2}$ and $\mu_{2}$ but it does not depend neither on $\lambda_{1}$ nor on $\mu_{1}$. Therefore, we assume that $\left|\mu_{1}\right|$ is small enough so that

$$
\tilde{c}\left(\mu_{1}^{2}+\left|\mu_{1}\right|\right)<c \min \left\{1, \lambda_{2}\right\} .
$$

Because $\lambda_{2}>0$ (according to (5.1)), the set of $\mu_{1}$ satisfying (5.37) is not empty and it is reduced to the one defined by a smallness condition of the form (3.8), for $j=1$ and $\mu_{1}^{0}=\frac{c}{\tilde{c}} \min \left\{1, \lambda_{2}\right\}$. Then 
(5.34) and $F \sim E$ imply that, for some positive constant $c_{1}$,

$$
F^{\prime}(t) \leq-c_{1} F(t)+\left(\frac{\rho_{1} k_{2}}{k_{1}}-\rho_{2}\right) \int_{0}^{L} \varphi_{t} \psi_{x t} \mathrm{~d} x .
$$

Because the last term of (5.25) vanishes (thanks to (1.4)), than (5.25) leads to (4.11), and then (3.3) is deduced as in the previous section.

Case 2: the boundary conditions (1.3) hold,

$$
\left|\mu_{1}\right|<\lambda_{1} \text { and }\left|\mu_{2}\right| \geq \lambda_{2} \text {. }
$$

Similarly to [3], we consider the following lemma:

Lemma 5.8: The functional

$$
J_{1}(t)=-\rho_{2} \int_{0}^{L} \psi_{t}\left(\varphi_{x}+\psi\right) \mathrm{d} x-\frac{k_{2} \rho_{1}}{k_{1}} \int_{0}^{L} \psi_{x} \varphi_{t} \mathrm{~d} x
$$

satisfies, for any $\delta>0$,

$$
\begin{aligned}
J_{1}^{\prime}(t) \leq & \left(k_{1}+\delta\right) \int_{0}^{L}\left(\varphi_{x}+\psi\right)^{2} \mathrm{~d} x-\rho_{2} \int_{0}^{L} \psi_{t}^{2} \mathrm{~d} x+\delta \int_{0}^{L} \psi_{x}^{2} \mathrm{~d} x+\left(\rho_{2}-\frac{k_{2} \rho_{1}}{k_{1}}\right) \int_{0}^{L} \varphi_{t} \psi_{x t} \mathrm{~d} x \\
& +c_{\delta} \int_{0}^{L}\left(\lambda_{1}^{2} \varphi_{t}^{2}+\lambda_{2}^{2} \psi_{t}^{2}+\mu_{1}^{2} \varphi_{t}^{2}\left(x, t-\tau_{1}\right)+\mu_{2}^{2} \psi_{t}^{2}\left(x, t-\tau_{2}\right)\right) \mathrm{d} x
\end{aligned}
$$

Proof: Using equations in (1.1) and the boundary conditions (1.3), we have

$$
\begin{aligned}
J_{1}^{\prime}(t)= & k_{1} \int_{0}^{L}\left(\varphi_{x}+\psi\right)^{2} \mathrm{~d} x-\rho_{2} \int_{0}^{L} \psi_{t}^{2} \mathrm{~d} x+\left(\rho_{2}-\frac{k_{2} \rho_{1}}{k_{1}}\right) \int_{0}^{L} \varphi_{t} \psi_{x t} \mathrm{~d} x \\
& +\int_{0}^{L}\left(\varphi_{x}+\psi\right)\left(\lambda_{2} \psi_{t}+\mu_{2} \psi_{t}\left(x, t-\tau_{2}\right)\right) \mathrm{d} x+\frac{k_{2}}{k_{1}} \int_{0}^{L} \psi_{x}\left(\lambda_{1} \varphi_{t}+\mu_{1} \varphi_{t}\left(x, t-\tau_{1}\right)\right) \mathrm{d} x .
\end{aligned}
$$

Arguing as for (5.27), we get (5.27).

Lemma 5.9: Let consider the functionals

$$
w(x, t)=\int_{0}^{x} \psi(y, t) \mathrm{d} y
$$

and

$$
J_{2}(t)=\int_{0}^{L}\left(\rho_{1} \varphi \varphi_{t}+\rho_{1} w \varphi_{t}+\frac{\lambda_{1}}{2} \varphi^{2}\right) \mathrm{d} x .
$$

Then, for any $\epsilon, \delta>0$,

$$
\begin{aligned}
J_{2}^{\prime}(t) \leq & -k_{1} \int_{0}^{L}\left(\varphi_{x}+\psi\right)^{2} \mathrm{~d} x+\left(\rho_{1}+\frac{c}{\epsilon}\right) \int_{0}^{L} \varphi_{t}^{2} \mathrm{~d} x+\epsilon \int_{0}^{L} \psi_{t}^{2} \mathrm{~d} x \\
& +\delta \int_{0}^{L}\left(\varphi_{x}^{2}+\psi_{x}^{2}\right) \mathrm{d} x+c_{\delta} \int_{0}^{L}\left(\lambda_{1}^{2} \varphi_{t}^{2}+\mu_{1}^{2} \varphi_{t}^{2}\left(x, t-\tau_{1}\right)\right) \mathrm{d} x .
\end{aligned}
$$

Proof: First, we have $w(0, t)=w(L, t)=0$ thanks to (2.17) (remeber that, in case (1.3), $\psi$ plays the role of $\tilde{\psi}$ ). Then (5.12) and (5.13) hold (as for (5.11)). Exploiting the first equation in (1.1), integrating 
by parts, using (5.12), (5.13), and the boundary conditions (1.3), and arguing as for (5.14), we find (5.29).

Now, for $N_{1}, N_{2}, N_{3}, N_{4}>0$, let

$$
F=N_{1} E+N_{2}\left(I_{1}+I_{2}\right)+N_{3} J+N_{4} J_{2}+J_{1}
$$

By combining (4.2), (4.4), (4.6), (5.27), and (5.29), and using (5.9), we obtain

$$
\begin{aligned}
F^{\prime}(t) \leq & -\left(k_{2} N_{3}-\delta\left(c N_{3}+c N_{4}+1\right)\right) \int_{0}^{L} \psi_{x}^{2} \mathrm{~d} x-\left(\left(1-N_{3}\right) \rho_{2}-\epsilon N_{4}\right) \int_{0}^{L} \psi_{t}^{2} \mathrm{~d} x \\
& +N_{1} E^{\prime}(t)+\left(N_{3} \rho_{1}+N_{4}\left(\rho_{1}+\frac{c}{\epsilon}\right)+\frac{\xi_{1} N_{2}}{\tau_{1}}\right) \int_{0}^{L} \varphi_{t}^{2} \mathrm{~d} x \\
& -\left(\left(N_{3}+N_{4}-1\right) k_{1}-\delta\left(2 N_{3}+2 N_{4}+1\right)\right) \int_{0}^{L}\left(\varphi_{x}+\psi\right)^{2} \mathrm{~d} x \\
& -2 N_{2} \int_{0}^{L} \int_{0}^{1}\left(\xi_{1} e^{-2 \tau_{1}} \varphi_{t}^{2}\left(x, t-\tau_{1} p\right)+\xi_{2} e^{-2 \tau_{2}} \psi_{t}^{2}\left(x, t-\tau_{2} p\right)\right) d p \mathrm{~d} x \\
& +\frac{\xi_{2} N_{2}}{\tau_{2}} \int_{0}^{L} \psi_{t}^{2} \mathrm{~d} x+\left(c_{\delta} \mu_{1}^{2}\left(N_{3}+N_{4}+1\right)-\frac{\xi_{1} e^{-2 \tau_{1}}}{\tau_{1}} N_{2}\right) \int_{0}^{L} \varphi_{t}^{2}\left(x, t-\tau_{1}\right) \mathrm{d} x \\
& +\left(c_{\delta} \mu_{2}^{2}\left(N_{3}+1\right)-\frac{\xi_{2} e^{-2 \tau_{2}}}{\tau_{2}} N_{2}\right) \int_{0}^{L} \psi_{t}^{2}\left(x, t-\tau_{2}\right) \mathrm{d} x \\
& +c_{\delta} \int_{0}^{L}\left(\lambda_{1}^{2}\left(N_{4}+1\right) \varphi_{t}^{2}+\lambda_{2}^{2} \psi_{t}^{2}\right) \mathrm{d} x+\left(\rho_{2}-\frac{\rho_{1} k_{2}}{k_{1}}\right) \int_{0}^{L} \varphi_{t} \psi_{x t} \mathrm{~d} x
\end{aligned}
$$

At this point, we choose

$$
N_{3}=\frac{1}{2}, \quad N_{4}=1+\frac{1}{\rho_{1}} \quad \text { and } \quad \epsilon=\frac{1}{N_{4}^{2}}
$$

then $\delta>0$ small enough so that

$$
\delta<\min \left\{\frac{k_{2} N_{3}}{c N_{3}+c N_{4}+1}, \frac{\left(N_{3}+N_{4}-1\right) k_{1}}{2 N_{3}+2 N_{4}+1}\right\} .
$$

Next, we pick $N_{2}>0$ large enough so that

$$
c_{\delta} \mu_{1}^{2}\left(N_{3}+N_{4}+1\right)-\frac{\xi_{1} e^{-2 \tau_{1}}}{\tau_{1}} N_{2} \leq 0 \quad \text { and } \quad c_{\delta} \mu_{2}^{2}\left(N_{3}+1\right)-\frac{\xi_{2} e^{-2 \tau_{2}}}{\tau_{2}} N_{2} \leq 0 .
$$

Notice that, according to (2.11), if $\mu_{j}=0$, then $\xi_{j}=0$. Otherwise, in virtue of (2.11) and (5.26), $\xi_{1}=\tau_{1} \lambda_{1}$ and $\xi_{2}=\tau_{2}\left|\mu_{2}\right|$. So $N_{2}$ exists and can be taken in the form

$$
N_{2}=c\left(\left|\mu_{2}\right|+\lambda_{1}\right)
$$

From the choice (5.32), the definition of $E$ and the fact that $\lambda_{2} \leq\left|\mu_{2}\right|$, we deduce from (5.31) that

$$
\begin{aligned}
F^{\prime}(t) \leq & -c \min \left\{1,\left|\mu_{2}\right|+\lambda_{1}\right\} E(t)+N_{1} E^{\prime}(t)+c\left(\mu_{2}^{2}+\lambda_{1}\left|\mu_{2}\right|\right) \int_{0}^{L} \psi_{t}^{2} \mathrm{~d} x \\
& +c\left(\lambda_{1}^{2}+\lambda_{1}\left|\mu_{2}\right|+1\right) \int_{0}^{L} \varphi_{t}^{2} \mathrm{~d} x+\left(\rho_{2}-\frac{\rho_{1} k_{2}}{k_{1}}\right) \int_{0}^{L} \varphi_{t} \psi_{x t} \mathrm{~d} x .
\end{aligned}
$$


Hence, according to (3.10) and (5.26), we have

$$
E^{\prime}(t) \leq \int_{0}^{L}\left(\frac{\left|\mu_{1}\right|-\lambda_{1}}{2} \varphi_{t}^{2}+\left(\left|\mu_{2}\right|-\lambda_{2}\right) \psi_{t}^{2}\right) \mathrm{d} x .
$$

By combining (5.35) and (5.34), we get $\left(\min \left\{1, \lambda_{1}\right\} \leq \min \left\{1,\left|\mu_{2}\right|+\lambda_{1}\right\}\right)$

$$
\begin{aligned}
F^{\prime}(t) \leq & -c \min \left\{1, \lambda_{1}\right\} E(t)+\left(\rho_{2}-\frac{\rho_{1} k_{2}}{k_{1}}\right) \int_{0}^{L} \varphi_{t} \psi_{x t} \mathrm{~d} x \\
& +\left(c\left(\mu_{2}^{2}+\lambda_{1}\left|\mu_{2}\right|\right)+N_{1}\left(\left|\mu_{2}\right|-\lambda_{2}\right)\right) \int_{0}^{L} \psi_{t}^{2} \mathrm{~d} x \\
& +\left(c\left(\lambda_{1}^{2}+\lambda_{1}\left|\mu_{2}\right|+1\right)+\frac{N_{1}}{2}\left(\left|\mu_{1}\right|-\lambda_{1}\right)\right) \int_{0}^{L} \varphi_{t}^{2} \mathrm{~d} x .
\end{aligned}
$$

On the other hand, by definition of the functionals $J, I_{1}, I_{2}, J_{1}, J_{2}$ and $E$ (notice that $\lambda_{2} \leq\left|\mu_{2}\right|$ ), we have

$$
\left|N_{2}\left(I_{1}+I_{2}\right)+N_{3} J+N_{4} J_{2}+J_{1}\right| \leq c\left(\left|\mu_{2}\right|+\lambda_{1}+1\right) E,
$$

which implies that

$$
\left(N_{1}-c\left(\left|\mu_{2}\right|+\lambda_{1}+1\right)\right) E \leq F \leq\left(N_{1}+c\left(\left|\mu_{2}\right|+\lambda_{1}+1\right)\right) E .
$$

Then we choose $N_{1}$ large enough such that

$$
c\left(\lambda_{1}^{2}+\lambda_{1}\left|\mu_{2}\right|+1\right)+\frac{N_{1}}{2}\left(\left|\mu_{1}\right|-\lambda_{1}\right) \leq 0 \quad \text { and } \quad N_{1}>c\left(\left|\mu_{2}\right|+\lambda_{1}+1\right) .
$$

Because $\left|\mu_{1}\right|<\lambda_{1}$, then $N_{1}$ exists and can be taken in the form

$$
N_{1}=c\left(\frac{\lambda_{1}^{2}+\lambda_{1}\left|\mu_{2}\right|+1}{\lambda_{1}-\left|\mu_{1}\right|}+\left|\mu_{2}\right|+\lambda_{1}+1\right),
$$

so the last term in (5.35) is non-positive and $F \sim E$. In addition, using the definition of $E$, we deduce from (5.35) and (5.36) that

$$
F^{\prime}(t) \leq-c \min \left\{1, \lambda_{1}\right\} E(t)+\tilde{c}\left(\mu_{2}^{2}+\left|\mu_{2}\right|\right) E(t)+\left(\rho_{2}-\frac{\rho_{1} k_{2}}{k_{1}}\right) \int_{0}^{L} \varphi_{t} \psi_{x t} \mathrm{~d} x,
$$

where $\tilde{c}$ is a positive constant which depends on $\lambda_{1}$ and $\mu_{1}$ but it does not depend neither on $\lambda_{2}$ nor on $\mu_{2}$. Therefore, we assume that $\left|\mu_{2}\right|$ is small enough so that

$$
\tilde{c}\left(\mu_{2}^{2}+\left|\mu_{2}\right|\right)<c \min \left\{1, \lambda_{1}\right\}
$$

Because $\lambda_{1}>0$ (according to (5.26)), the set of $\mu_{2}$ satisfying (5.39) is not empty and it is reduced to the one defined by a smallness condition of the form (3.8), for $j=2$ and $\mu_{2}^{0}=\frac{c}{\tilde{c}} \min \left\{1, \lambda_{1}\right\}$. Then (5.37) and $F \sim E$ imply that, for some positive constant $c_{1}$,

$$
F^{\prime}(t) \leq-c_{1} F(t)+\left(\rho_{2}-\frac{\rho_{1} k_{2}}{k_{1}}\right) \int_{0}^{L} \varphi_{t} \psi_{x t} \mathrm{~d} x
$$

The end of the proof is the same as in the Case 1. 


\section{Proof of Theorem 3.3}

Assume that (1.4) does not hold, (3.4) or (3.5) holds and let $\mathscr{U}_{0} \in D(\mathscr{A})$. As in Theorem 3.2, we distinguish two cases.

Case 1: the boundary conditions (1.2) or (1.3) hold,

$$
\left|\mu_{1}\right|=\lambda_{1} \text { and }\left|\mu_{2}\right|<\lambda_{2} \text {. }
$$

We will estimate the last term in (5.25) using the system (6) resulting from differentiating (1.1), (1.2), and (1.3) with respect to time; that is

$$
\left\{\begin{array}{l}
\rho_{1} \varphi_{t t t}(x, t)-k_{1}\left(\varphi_{x t}+\psi_{t}\right)_{x}(x, t)+\lambda_{1} \varphi_{t t}(x, t)+\mu_{1} \varphi_{t t}\left(x, t-\tau_{1}\right)=0, \\
\rho_{2} \psi_{t t t}(x, t)-k_{2} \psi_{x x t}(x, t)+k_{1}\left(\varphi_{x t}+\psi_{t}\right)(x, t)+\lambda_{2} \psi_{t t}(x, t)+\mu_{2} \psi_{t t}\left(x, t-\tau_{2}\right)=0
\end{array}\right.
$$

with Dirichlet-Dirichlet boundary conditions

$$
\varphi_{t}(0, t)=\varphi_{t}(L, t)=\psi_{t}(0, t)=\psi_{t}(L, t)=0
$$

or Dirichlet-Neumann boundary conditions

$$
\varphi_{t}(0, t)=\varphi_{t}(L, t)=\psi_{x t}(0, t)=\psi_{x t}(L, t)=0 .
$$

System (6) with (6) or (6) is well posed for initial data $\mathscr{U}_{0} \in D(\mathscr{A})$ (see Theorem 2.2). Let $E_{2}$ be the second-order energy (the energy of (6)) defined by $E_{2}(t)=E\left(\mathscr{U}_{t}(t)\right)$, where $E(\mathscr{U}(t))=E(t)$ and $E$ is defined by (3.1)). As for (3.10) and according to (6), a simple calculation implies that

$$
E_{2}^{\prime}(t) \leq d_{2} \int_{0}^{L} \psi_{t t}^{2} \mathrm{~d} x
$$

so, as $E$, also $E_{2}$ is non-increasing.

Lemma 6.1: For any $\epsilon>0$, there exists a positive constant $\alpha_{\epsilon}$ such that

$$
\left(\frac{\rho_{1} k_{2}}{k_{1}}-\rho_{2}\right) \int_{S}^{T} \int_{0}^{L} \varphi_{x t} \psi_{t} \mathrm{~d} x \mathrm{~d} t \leq \epsilon \int_{S}^{T} E(t) \mathrm{d} t+\alpha_{\epsilon}\left(E(S)+E_{2}(S)\right), \quad \forall T \geq S \geq 0 .
$$

Proof: By integration with respect to $t$, we get

$$
\int_{S}^{T} \int_{0}^{L} \varphi_{x t} \psi_{t} \mathrm{~d} x \mathrm{~d} t=\left[\int_{0}^{L} \varphi_{x} \psi_{t} \mathrm{~d} x\right]_{S}^{T}-\int_{S}^{T} \int_{0}^{L} \varphi_{x} \psi_{t t} \mathrm{~d} x \mathrm{~d} t
$$

Moreover, using the definition of $E$ and its non-increasingness, we find

$$
\left|\left(\frac{\rho_{1} k_{2}}{k_{1}}-\rho_{2}\right) \int_{0}^{L} \varphi_{x} \psi_{t} \mathrm{~d} x\right| \leq c E(t) \leq c E(S), \quad \forall 0 \leq S \leq t .
$$

Thus, from (6) we have

$$
\left(\frac{\rho_{1} k_{2}}{k_{1}}-\rho_{2}\right) \int_{S}^{T} \int_{0}^{L} \varphi_{x t} \psi_{t} \mathrm{~d} x \mathrm{~d} t \leq c E(S)+c \int_{S}^{T} \int_{0}^{L}\left|\varphi_{x}\right|\left|\psi_{t t}\right| \mathrm{d} x \mathrm{~d} t, \quad \forall T \geq S \geq 0
$$


On the other hand, because $d_{2}<0$, (6) leads to

$$
\int_{0}^{L} \psi_{t t}^{2} \mathrm{~d} x \leq \frac{1}{d_{2}} E_{2}^{\prime}(t)
$$

Then, using Young's inequality, we estimate the last integral in (6) as follows:

$$
\begin{aligned}
c \int_{S}^{T} \int_{0}^{L}\left|\varphi_{x}\right|\left|\psi_{t t}\right| \mathrm{d} x \mathrm{~d} t & \leq \epsilon \int_{S}^{T} E(t) \mathrm{d} t-\frac{c_{\epsilon}}{d_{2}} \int_{S}^{T} E_{2}^{\prime}(t) \mathrm{d} t \\
& \leq \epsilon \int_{S}^{T} E(t) \mathrm{d} t+\frac{c_{\epsilon}}{d_{2}} E_{2}(S), \quad \forall T \geq S \geq 0 .
\end{aligned}
$$

Inserting this inequality into (6), we get (6.1) with $\alpha_{\epsilon}=\max \left\{c, \frac{c_{\epsilon}}{d_{2}}\right\}$.

Now, exploiting (5.25) and (6.1), using the property $F \sim E$, and choosing $\epsilon>0$ small enough, we get, for some positive constants $\alpha_{1}$ and $\alpha_{2}$,

$$
\int_{S}^{T} F^{\prime}(t) \mathrm{d} t \leq-\alpha_{1} \int_{S}^{T} E(t) \mathrm{d} t+\alpha_{2}\left(E(S)+E_{2}(S)\right), \quad \forall T \geq S \geq 0 .
$$

By combining (6) and the property $F \sim E$, we deduce that, for some positive constant $\alpha_{3}$,

$$
\int_{S}^{T} E(t) \mathrm{d} t \leq \alpha_{3}\left(E(S)+E_{2}(S)\right), \quad \forall T \geq S \geq 0 .
$$

Choosing $S=0$ in (6) and using the fact that $E$ is non-increasing, we get

$$
E(T) T \leq \int_{0}^{T} E(t) \mathrm{d} t \leq \alpha_{3}\left(E(0)+E_{2}(0)\right):=c_{1}, \quad \forall T \geq 0,
$$

which gives (3.9).

Case 2: the boundary conditions (1.3) hold,

$$
\left|\mu_{1}\right|<\lambda_{1} \quad \text { and } \quad\left|\mu_{2}\right|=\lambda_{2} \text {. }
$$

According to (6), a simple calculation implies that, as for (6),

$$
E_{2}^{\prime}(t) \leq d_{1} \int_{0}^{L} \varphi_{t t}^{2} \mathrm{~d} x
$$

Lemma 6.2: For any $\epsilon>0$, there exists a positive constant $\alpha_{\epsilon}$ such that, for all $T \geq S \geq 0$,

$$
\left(\rho_{2}-\frac{\rho_{1} k_{2}}{k_{1}}\right) \int_{S}^{T} \int_{0}^{L} \varphi_{x t} \psi_{t} \mathrm{~d} x \mathrm{~d} t \leq \epsilon \int_{S}^{T} E(t) \mathrm{d} t+\alpha_{\epsilon}\left(E(S)+E_{2}(S)\right) .
$$

Proof: By integration with respect to $x$ and $t$, and using the boundary condition (1.3), we get

$$
\int_{S}^{T} \int_{0}^{L} \varphi_{x t} \psi_{t} \mathrm{~d} x \mathrm{~d} t=-\int_{S}^{T} \int_{0}^{L} \varphi_{t} \psi_{x t} \mathrm{~d} x \mathrm{~d} t=-\left[\int_{0}^{L} \varphi_{t} \psi_{x} \mathrm{~d} x\right]_{S}^{T}+\int_{S}^{T} \int_{0}^{L} \varphi_{t t} \psi_{x} \mathrm{~d} x \mathrm{~d} t
$$

Using (6), the proof of (6.2) can be finished as for (6.1).

Exploiting (5.39) and (6.2), the proof of (3.9) can be ended as in the Case 1. 


\section{Concluding remarks}

In this section, we conclude with some remarks and list some open questions for the interested reader. Remark 7.1: When (3.6) or [(1.3) and (3.7)] hold, we can take $\left[\lambda_{1}=0\right.$ and $\left.\mu_{1} \mu_{2} \neq 0\right]$ or $\left[\lambda_{2}=0\right.$ and $\left.\mu_{1} \mu_{2} \neq 0\right]$ or $\left[\lambda_{1}=\mu_{2}=0\right.$ and $\left.\lambda_{2} \mu_{1} \neq 0\right]$ or $\left[\lambda_{2}=\mu_{1}=0\right.$ and $\left.\lambda_{1} \mu_{2} \neq 0\right]$. These cases show that, provided that (1.4) is satisfied, the exponential stability (3.3) of (1.1) holds also under one internal frictional damping and two discrete time delays or under one internal frictional damping and one discrete time delay not considered on the same equation.

Remark 7.2: Our results remain true if we consider Timoshenko-type systems with variables coeffiecients $\lambda_{j}(x)$ and $\mu_{j}(x)$ satisfying some smoothness and smallness conditions by modifying the operators and Lyapunov functionals considered in our proof (see [9] in case $\lambda_{2}=\mu_{2}=0$ ).

Remark 7.3: We can consider distributed time delays

$$
\int_{O}^{+\infty} h_{1}(s) \varphi_{t}(x, t-s) \mathrm{d} s \text { and } \int_{O}^{+\infty} h_{2}(s) \psi_{t}(x, t-s) \mathrm{d} s
$$

instead of the discrete ones $\mu_{1} \varphi_{t}\left(x, t-\tau_{1}\right)$ and $\mu_{2} \psi_{t}\left(x, t-\tau_{2}\right)$, respectively, in both first two equations in (1.1) or in one of them, where $h_{1}, h_{2}: \mathbb{R}^{+} \rightarrow \mathbb{R}$ are some given functions (see [8] in case $\left.\lambda_{1}=\mu_{1}=0\right)$.

Remark 7.4: The estimate (3.9) can be generalized by proving that, for any $n \in \mathbb{N}^{*}$ and $\mathscr{U}_{0} \in$ $D\left(\mathscr{A}^{n}\right)$, there exists a positive constant $c_{n}>0$ such that

$$
E(t) \leq \frac{c_{n}}{t^{n}}, \quad \forall t>0
$$

(see [9] in case $\lambda_{2}=\mu_{2}=0$ ).

Remark 7.5: Our stability results can be generalized to the case where, for some given functions $\phi_{1}, \phi_{2}: \mathbb{R} \rightarrow \mathbb{R}$, the linear frictional dampings $\lambda_{1} \varphi_{t}$ and $\lambda_{2} \psi_{t}$ are replaced by the nonlinear ones $\phi_{1}\left(\varphi_{t}\right)$ and $\phi_{2}\left(\psi_{t}\right)$, respectively.

Remark 7.6: We do not know if (1.1) is stable when (1.4) does not hold, and (3.6) or (3.7) holds. Similarly, we do not know if (1.1) is stable when [(1.2) and (3.5)] or [(1.2) and (3.7)] hold. On the other hand, the stability of (1.1) is an open question when (3.4) or (3.5) or (3.6) or (3.7) holds but $\left|\mu_{j}\right|$ is not small enough.

Remark 7.7: The stability of (1.1) with (1.2) or (1.3) when

$$
\left|\mu_{1}\right| \geq \lambda_{1} \text { and }\left|\mu_{2}\right| \geq \lambda_{2}
$$

seems not being satisfied. It was proved in [34] that the stability of the wave equation with internal frictional damping and discrete time delay does not hold (even for small time delay) when the weight of the delay is bigger than the one of the damping.

Remark 7.8: It will be interesting to extend our results to the following system:

$$
\left\{\begin{array}{l}
\rho_{1} \varphi_{t t}(x, t)-k_{1}\left(\varphi_{x}+\psi\right)_{x}(x, t)+\mu_{1} \varphi_{t}\left(x, t-\tau_{1}\right)=0, \\
\rho_{2} \psi_{t t}(x, t)-k_{2} \psi_{x x}(x, t)+k_{1}\left(\varphi_{x}+\psi\right)(x, t)+\mu_{2} \psi_{t}\left(x, t-\tau_{2}\right)=0, \\
\varphi(x, 0)=\varphi_{0}(x), \varphi_{t}(x, 0)=\varphi_{1}(x), \\
\psi(x, 0)=\psi_{0}(x), \psi_{t}(x, 0)=\psi_{1}(x), \\
\varphi_{t}\left(x,-\tau_{1} \rho\right)=f_{1}\left(x,-\tau_{1} \rho\right), \psi_{t}\left(x,-\tau_{2} \rho\right)=f_{2}\left(x,-\tau_{2} \rho\right)
\end{array}\right.
$$


under the following boundary conditions:

$$
\left\{\begin{array}{l}
\left(\varphi_{x}+\psi\right)(L, t)+\lambda_{1} \varphi_{t}(L, t)=0 \\
\psi_{x}(L, t)+\lambda_{2} \psi_{t}(L, t)=0, \\
\varphi(0, t)=\psi(0, t)=0 .
\end{array}\right.
$$

\section{Acknowledgements}

This work was initiated during the visit of the first author to the University of Sharjah, UAE, in April 2014. The first author wishes to thank this university for its kind hospitality.

\section{Disclosure statement}

No potential conflict of interest was reported by the authors.

\section{References}

[1] Almeida Júnior DS, Santos ML, Muñoz Rivera JE. Stability to weakly dissipative Timoshenko systems. Math. Meth. Appl. Sci. 2013;36:1965-1976.

[2] Almeida Júnior DS, Santos ML, Muñoz Rivera JE. Stability to 1-D thermoelastic Timoshenko beam acting on shear force. Z. Angew. Math. Phys. 2014;65:1233-1249.

[3] Ammar-Khodja F, Benabdallah A, Muñoz Rivera JE, et al. Energy decay for Timoshenko systems of memory type. J. Differ. Equ. 2003;194:82-115.

[4] Apalara TA, Messaoudi SA, Mustafa MI. Energy decay in Thermoelasticity type III with viscoelastic damping and delay term. Elec. J. Diff. Equa. 2012;128:1-15.

[5] Benaissa A, Benaissa AK, Messaoudi SA. Global existence and energy decay of solutions for the wave equation with a time varying delay term in the weakly nonlinear internal feedbacks. J. Math. Phys. 2012;53:123514.

[6] Datko R, Lagnese J, Polis MP. An example on the effect of time delays in boundary feedback stabilization of wave equations. SIAM J. Control Optim. 1986;1:152-156.

[7] Guesmia A. On the stabilization for Timoshenko system with past history and frictional damping controls. Palestine J. Math. 2013;2:187-214.

[8] Guesmia A. Some well-posedness and general stability results in Timoshenko systems with infinite memory and distributed time delay. J. Math. Phys. 2014;55:1-40.

[9] Guesmia A. Well-posedness and energy decay for Timoshenko systems with discrete time delay under frictional damping and/or infinite memory in the displacement. J. Control Evol. Equ. submitted.

[10] Guesmia A, Messaoudi SA. On the control of solutions of a viscoelastic equation. Appl. Math. Comput. 2008;206:589-597.

[11] Guesmia A, Messaoudi SA. General energy decay estimates of Timoshenko systems with frictional versus viscoelastic damping. Math. Meth. Appl. Sci. 2009;32:2102-2122.

[12] Guesmia A, Messaoudi SA. On the stabilization of Timoshenko systems with memory and different speeds of wave propagation. Appl. Math. Comput. 2013;219:9424-9437.

[13] Guesmia A, Messaoudi SA. A general stability result in a Timoshenko system with infinite memory: a new approach. Math. Meth. Appl. Sci. 2014;37:384-392.

[14] Guesmia A, Messaoudi SA. Some stability results for Timoshenko systems with cooperative frictional and infinitememory dampings in the displacement. Acta Math. Sci. 2016;36:1-33.

[15] Guesmia A, Messaoudi SA, Soufyane A. Stabilization of a linear Timoshenko system with infinite history and applications to the Timoshenko-Heat systems. Electron. J. Differ. Equ. 2012;2012:1-45.

[16] Guesmia A, Messaoudi S, Wehbe A. Uniform decay in mildly damped Timoshenko systems with non-equal wave speed propagation. Dyn. Syst. Appl. 2012;21:133-146.

[17] Guesmia A, Tatar N. Some well-posedness and stability results for abstract hyperbolic equations with infinite memory and distributed time delay. Commun. Pure Appl. Anal, 2015;14:457-491.

[18] Kafini M, Messaoudi SA, Mustafa MI. Energy decay result in a Timoshenko-type system of thermoelasticity of type III with distributive delay. J. Math. Phys. 2013;54:101503.

[19] Kafini M, Messaoudi SA, Mustafa MI. Energy decay rates for a Timoshenko-type system of thermoelasticity of type III with constant delay. Appl. Anal. 2014;63:1201-1216.

[20] Kim JU, Renardy Y. Boundary control of the Timoshenko beam. SIAM J. Control Optim. 1987;25:1417-1429.

[21] Kirane M, Said-Houari B, Anwar MN. Stability result for the Timoshenko system with a time-varying delay term in the internal feedbacks. Commun. Pure Appl. Anal. 2011;10:667-686. 
[22] Komornik V. Exact controllability and stabilization. The multiplier method. Paris: Masson-John Wiley; 1994.

[23] Messaoudi SA, Apalara TA. Asymptotic stability of thermoelasticity type III with delay term and infinite memory. IMA J. Math. Control Inf. 2015;32:75-95.

[24] Messaoudi SA, Michael P, Said-Houari B. Nonlinear damped Timoshenko systems with second: global existence and exponential stability. Math. Meth. Appl. Sci. 2009;32:505-534.

[25] Messaoudi SA, Mustafa MI. On the internal and boundary stabilization of Timoshenko beams. Nonlinear Differ. Equ. Appl. 2008;15:655-671.

[26] Messaoudi SA, Mustafa MI. On the stabilization of the Timoshenko system by a weak nonlinear dissipation. Math. Methods Appl. Sci. 2009;32:454-469.

[27] Messaoudi SA, Mustafa MI. A stability result in a memory-type Timoshenko system. Dyn. Syst. Appl. 2009;18:457468.

[28] Messaoudi SA. and Said-Houari Belkacem, Uniform decay in a Timoshenko-type system with past history. J. Math. Anal. Appl. 2009;360:459-475.

[29] Muñoz Rivera JE, Fernández Sare HD. Stability of Timoshenko systems with past history. J. Math. Anal. Appl. 2008;339:482-502.

[30] Muñoz Rivera JE, Racke R. Mildly dissipative nonlinear Timoshenko systems-global existence and exponential stability. J. Math. Anal. Appl. 2002;276:248-278.

[31] Muñoz Rivera JE, Racke R. Global stability for damped Timoshenko systems. Discrete Contin. Dyn. Syst. 2003;9:1625-1639.

[32] Muñoz Rivera JE, Racke R. Timoshenko systems with indefinite damping. J. Math. Anal. Appl. 2008;341:10681083.

[33] Mustafa MI, Messaoudi SA. General energy decay rates for a weakly damped Timoshenko system. Dyn.Control Syst. 2010;16:211-226.

[34] Nicaise S, Pignotti C. Stability and instability results of the wave equation with a delay term in the boundary or internal feedbacks. SIAM J. Control Optim. 2006;5:1561-1585.

[35] Nicaise S, Pignotti C. Stabilization of the wave equation with boundary or internal distributed delay. Differ. Integr. Equ. 2008;9-10:935-958.

[36] Nicaise S, Pignotti C. Interior feedback stabilization of wave equations with time dependent delay. Electron. J. Differ. Equ. 2011;41:1-20.

[37] Nicaise S, Pignotti C, Valein J. Exponential stability of the wave equation with boundary time-varying delay. Disc. Contin. Dyn. Syst. Ser. S. 2011;3:693-722.

[38] Nicaise S, Valein J, Fridman E. Stability of the heat and of the wave equations with boundary time-varying delays. Disc. Cont. Dyna. Syst. Series S. 2009;2:559-581.

[39] Pazy A. Semigroups of linear operators and applications to partial differential equations. New York (NY): Springer-Verlag; 1983.

[40] Racke R, Said-Houari B. Global existence and decay property of the Timoshenko system in thermoelasticity with second sound. Nonlinear Analysis. 2012;75:4957-4973.

[41] Racke R, Said-Houari B. Decay rates and global existence for semilinear dissipative Timoshenko systems. Quart. Appl. Math. 2013;71:229-266.

[42] Raposo CA, Ferreira J, Santos ML, Castro NNO. Exponential stability for the Timoshenko system with two week dampings. Appl. Math. Letters. 2005;18:535-541.

[43] Said-Houari B. A stability result for a Timoshenko system with past history and a delay term in the internal feedback. Dyn. Syst. Appl. 2011;20:327-354.

[44] Said-Houari B, Kasimov A. Decay property of Timoshenko system in thermoelasticity. Math. Meth. Appl. Sci. 2012;35:314-333.

[45] Said-Houari B, Kasimov A. Damping by heat conduction in the Timoshenko system: Fourier and Cattaneo are the same. J. Diff. Equa. 2013;255:611-632.

[46] Said-Houari B, Laskri Y. A stability result of a Timoshenko system with a delay term in the internal feedback. Appl. Math. Comput. 2010;217:2857-2869.

[47] Said-Houari B, Soufyane A. Stability result of the Timoshenko system with delay and boundary feedback. IMA J. Math. Control Inf. 2012;29:383-398.

[48] Santos ML, Almeida Júnior DS, Muñoz Rivera JE. The stability number of the Timoshenko system with second sound. J. Differ. Equ. 2012;253:2715-2733.

[49] Soufyane A, Wehbe A. Uniform stabilization for the Timoshenko beam by a locally distributed damping. Elecron. J. Differ. Equ. 2003;29:1-14.

[50] Timoshenko S. On the correction for shear of the differential equation for transverse vibrations of prismaticbars. Philis. Mag. 1921;41:744-746. 